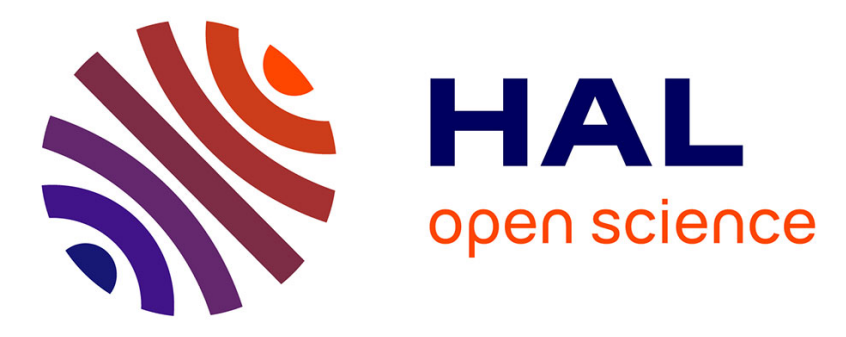

\title{
Some elements for a history of the dynamical systems theory
}

\author{
Christophe Letellier, Ralph Abraham, Dima L Shepelyansky, Otto E Rössler, \\ Philip Holmes, René Lozi, Leon Glass, Arkady Pikovsky, Lars F Olsen, Ichiro \\ Tsuda, et al.
}

\section{To cite this version:}

Christophe Letellier, Ralph Abraham, Dima L Shepelyansky, Otto E Rössler, Philip Holmes, et al.. Some elements for a history of the dynamical systems theory. Chaos: An Interdisciplinary Journal of Nonlinear Science, 2021, 31 (5), pp.053110. 10.1063/5.0047851 . hal-03230576

\section{HAL Id: hal-03230576 https://hal.science/hal-03230576}

Submitted on 20 May 2021

HAL is a multi-disciplinary open access archive for the deposit and dissemination of scientific research documents, whether they are published or not. The documents may come from teaching and research institutions in France or abroad, or from public or private research centers.
L'archive ouverte pluridisciplinaire HAL, est destinée au dépôt et à la diffusion de documents scientifiques de niveau recherche, publiés ou non, émanant des établissements d'enseignement et de recherche français ou étrangers, des laboratoires publics ou privés. 


\section{Some elements for a history of the dynamical systems theory}

Christophe Letellier, ${ }^{1}$ Ralph Abraham, ${ }^{2}$ Dima L. Shepelyansky, ${ }^{3}$ Otto E. Rössler, ${ }^{4}$ Philip Holmes, ${ }^{5}$ René Lozi, ${ }^{6}$

Leon Glass, ${ }^{7}$ Arkady Pikovsky, ${ }^{8}$ Lars F. Olsen, ${ }^{9}$ Ichiro Tsuda, ${ }^{10}$ Celso Grebogi, ${ }^{11}$ Ulrich Parlitz, ${ }^{12}$ Robert

Gilmore, ${ }^{13}$ Louis M. Pecora, ${ }^{14}$ and Thomas L. Carroll ${ }^{14}$

${ }^{1)}$ Normandie Université - CORIA, Campus Universitaire du Madrillet, F-76800 Saint-Etienne du Rouvray,

France.

${ }^{2)}$ University of California, Santa Cruz, CA 95064 USA

${ }^{3)}$ Laboratoire de Physique Théorique, IRSAMC, Université de Toulouse, CNRS, UPS, 31062 Toulouse,

France $^{\text {a) }}$

4) University of Tübingen, D-72076 Tübingen, Germany

${ }^{5)}$ Department of Mechanical and Aerospace Engineering and Program in Applied and Computational Mathematics,

Princeton University, Princeton, NJ 08544, USA

6) Université Côte d'Azur, CNRS, Laboratoire Jean Alexandre Dieudonné, F-06108 Nice,

France

7) Department of Physiology, McGill University, 3655 Promenade Sir William Osler, Montreal H3G 1Y6, Quebec, Canada

8) Institute of Physics and Astronomy, University of Potsdam, Karl-Liebknecht-Str. 24/25, 14476 Potsdam-Golm,

Germany

${ }^{9)}$ Institute of Biochemistry and Molecular Biology, University of Southern Denmark, Campusvej 55, DK-5230 Odense M, Denmark

${ }^{10)}$ Chubu University Academy of Emerging Sciences, Matumoto-cho 1200, Kasugai, Aichi 487-8501, Japan

${ }^{11)}$ Institute for Complex Systems and Mathematical Biology, King's College, University of Aberdeen, Aberdeen AB24 3UE, Scotland

${ }^{12)}$ Max Planck Institute for Dynamics and Self-Organization, Am Fassberg 17, 37077 Göttingen, Germany - Institute for the Dynamics of Complex Systems, University of Göttingen, Friedrich-Hund-Platz 1, 37077 Göttingen, Germany

${ }^{13)}$ Drexel University, Philadelphia, PA 19104 USA

${ }^{14)}$ Code 6392, U.S. Naval Research Laboratory, Washington, DC, 20375, USA 202-767-6002

(Dated: 3 May 2021)

Writing a history of a scientific theory is always difficult because it requires to focus on some key contributors and to "reconstruct" some supposed influences. In the 1970s emerged a new way of performing science under the name "chaos", combining the mathematics from the nonlinear dynamical systems theory and numerical simulations. To provide a direct testimony of how contributors can be influenced by other scientists or works, we here collected some writings about the early times of a few contributors to chaos theory. The purpose is to exhibit the diversity in the paths and to bring some elements - which were never published - illustrating the atmosphere of this period. Some peculiarities of chaos theory are also discussed.

Chaos is a word which is, in science, very often quickly associated with the overgeneralized butterfly effect, inherited from the title of a talk given in 1972 by Edward Lorenz, one of the great contributors to the so-called chaos theory. This theory is a branch of the nonlinear dynamical systems (NDS) theory which was boosted by Poincaré's works at the late 19th century. It was then further developed by many great mathematicians for few decades. In the 1960s, with the occurence of computers, chaos theory emerged as a new methodology which is neither "pure" mathematics nor disconnected from the strongly mathematical NDS theory. The scientists working on chaos constitute a very interdisciplinary community whose emergence is associated with a high rate of disciplinary migration. Some of its contributors describe here how this migration occured.

\footnotetext{
${ }^{a)}$ http://www.quantware.ups-tlse.fr/dima
}

\section{INTRODUCTION BY CHRISTOPHE LETELLIER}

Chaos emerged in the 1970s. In their contribution to the problem of turbulence, Ruelle and Takens introduced the concept of strange attractor, strange meaning neither a limit cycle nor a quasiperiodic motion. ${ }^{1}$ They associated turbulence with a "very complicated, irregular and chaotic" motion. A few years later the term chaos was used by $\mathrm{Li}$ and Yorke in a very suggestive title Period-3 implies chaos. ${ }^{2}$ Then Rössler used it systematically to designate the aperiodic behavior he was studying in the state space. ${ }^{3-6}$ With the word "chaos" as a banner, scientists paid attention to aperiodic solutions which were not quasi-periodic and characterized with concepts inherited from the early works by Poincare ${ }^{7,8}$ and Birkhoff ${ }^{9}$ which were synthetized in a masterpiece by Lorenz in $1963 .{ }^{10}$ A history of the dynamical systems theory and chaos was already provided by Aubin and Dahan Dalmedico, focusing on three important contributors from the 1960s (Smale, Lorenz and Ruelle). ${ }^{11}$ This field is polymorphic and many branches emerged in the 1960s, in mathematics with Thom and Smale, 
in plasma physics with Chirikov, in meteorology with Lorenz, in control theory with Mira and Gumowski,... and exploded in the 1970s. Depending on the field from which it emerged, the influences were not always the same. Browsing the list of quotations in pioneering papers does not always allow to reveal them as evidenced with Lorenz's paper in which the book by Nemytskii and Stepanov ${ }^{12}$ is quoted after the suggestion from a reviewer. ${ }^{13}$ It is therefore important to have access to direct recollections of contributors as published, for instance, by Abraham and Ueda. ${ }^{14}$

This paper is devoted to a few contributors who never wrote before about their early times in chaos. Some others were contacted but declined the invitation. All of them were asked to focus on their early times without any other indication. Various drafts were produced, inflating under some exchanges with Letellier who always asked for more details. Contributors were always left free to develop or not some points. No length limitation was imposed and final texts extend from 2 to 22 pages. Here only excerpts are selected by Letellier, focusing on the very early times. When possible, the context in which a notable result was obtained is exhibited. These excerpts may (should ?) be considered as biased, by contributor's views as well as by the "selection process" from the whole text. We do not pretend to provide an objective view of the history of chaos, but rather how each one remembers his history of chaos. One of the motivations - not clearly stated to the contributors - was to exhibit the plurality of the paths followed by some scientists who contributed to chaos theory as well as the recurrence of some influential works.

The subsequent part of this paper is organized as follows. Sections II to XIV are the excerpts of the contributions, ordered according to a chronological order of the key contributions. Section XV provides a short analysis and gives some conclusion.

\section{THE SMALE PROGRAM BY RALPH ABRAHAM}

Steve Smale finished his Ph.D. thesis in differential topology in 1956, working with Raoul Bott at the University of Michigan. At that time I was there in Ann Arbor, finishing my undergraduate program in Engineering Mathematics. I was introduced to differential topology in a course by Bott, on general relativity in 1960, working with Nathaniel Coburn.

Solomon Lefschetz began devoting half of every year to build up a graduate program in the mathematics department of the National Autonomous University of Mexico. He had become interested in the Russian literature on dynamical systems theory. ${ }^{15}$ Smale attended Lefschetz' summer conference in Mexico City. ${ }^{16}$ There he met René Thom, Morris Hirsch, and Elon Lima. Around 1958, Lima finished his Ph.D. thesis on topology with Edwin Spanier in Chicago, and introduced Smale to Mauricio Peixoto. Peixoto was a Brazilian student of Lefschetz in Princeton, 1958-59. His theorem on the structural stability of flows in two dimensions, ${ }^{17}$ was an early breakthrough in dynamical systems theory.

In 1960, I arrived at UC Berkeley which, suddenly had a brand new staff of mathematics professors and visitors.
Smale arrived along with Spanier (algebraic topology), ShingShen Chern (differential geometry), and Hirsch (differential topology) from Chicago, Thom (differential topology) from Paris, Chris Zeeman (topology, expositor of catastrophe theory) from Warwick, Peixoto from Rio, Bob Williams (knot theory), Dick Palais (nonlinear functional analysis) and others comprising a research group on dynamical systems theory based on differential topology. Hirsch (a student of Spanier) and I were among the newbies in this group. The Smale program was focused on the stable manifolds, structural stability, and conjugacy of diffeomorphisms. At this time we devoted much time reading and discussing the works of Poincaré and Birkhoff, especially concerning the stable curves of surface transformations and their transversal intersections.

Smale had proved the existence of stable and unstable manifolds, his first major result in this field. ${ }^{18} \mathrm{He}$ developed the horseshoe map, his second major result. After this publication Thom proved that transversal intersection of stable manifolds is a generic property of diffeomorphisms. ${ }^{19}$

Smale's program was boosted into orbit by his influential survey ${ }^{20}$ which set out its foundations: conjugacy of diffeomorphisms, fixed and periodic points, stable and generic properties, the nonwandering set, hyperbolic fixed points, stable manifolds, ... Already we find drawings of homoclinic intersections of stable and unstable manifolds for surface transformations, discovered by Poincaré and analyzed in detail by Birkhoff and Smith. ${ }^{21}$ Smale's ingenious simplification of the homoclinic tangle in the two-dimensional case, the horseshoe map, is shown in Fig. 1. Smale carefully credits his predecessors - Poincaré22, Birkhoff, ${ }^{23}$, Morse, ${ }^{24}$ Andronov and Pontrjagin, ${ }^{25}$ Thom, ${ }^{26}$ Elsgolts, ${ }^{27}$ Reeb, ${ }^{28}$ and Peixoto. ${ }^{29}$

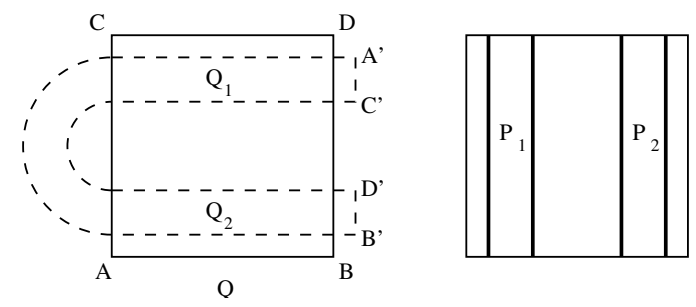

FIG. 1. Smale's horseshoe map. A global diffeomorphism maps the square $\mathrm{Q}$ into the region bounded by dotted lines with $G(\mathrm{~A})=\mathrm{A}^{\prime}$, etc. Each component $\mathrm{P}_{1}$ and $\mathrm{P}_{2}$ of $g^{-1}(g(\mathrm{Q}) \cap \mathrm{Q})$ is such that $g$ is a linear map with $g\left(\mathrm{P}_{i}\right)=\mathrm{Q}_{i}(i=1,2)$.

In 1962 I moved on to Columbia, and in 1964, to Princeton where Lefschetz still had huge influence. I was able to teach graduate courses and, with Jerry Marsden and Joel Robbin, I rewrote much of celestial mechanics with the new language and technology of global analysis. ${ }^{30}$ In another, I treated the transversality of stable manifolds in the global context of (infinite-dimensional) manifolds of mappings. ${ }^{31}$

Around 1966, I began to receive letters from René Thom in which he reported regular progress in his creation of catastrophe theory. In this simple context of gradient (non-chaotic) dynamical systems, he made crucial use of the language of attractors, basins, and bifurcations, which became fundamental in the further evolution of dynamical systems theory, and later, chaos theory. He popularized a style of application of 
these notions, introduced earlier by Poincaré and his Russian followers. ${ }^{32}$ The impact on the mathematical community was further facilitated by a series of exemplary articles by Zeeman. ${ }^{33}$

In 1968, a four-week conference on global analysis (July 126) was edited by Chern and Smale. ${ }^{34}$ This was the moment, I believe, at which our group finally became aware of the experimental work and simulations on chaotic attractors. Yoshisuke Ueda, discovered the first clearly chaotic attractor in analog simulation, the Japanese attractor, ${ }^{35}$ for which he accurately drew the homoclinic tangle of inset and outset curves for the forced Duffing equation

$$
\left\{\begin{array}{l}
\dot{x}=y \\
\dot{y}=\mu\left(1-\gamma x^{2}\right) y-x^{3}+B \cos v t
\end{array}\right.
$$

obtained at Kyoto University, on November, 1961 (Fig. 2), ${ }^{36}$ Edward Lorenz, discovered his chaotic attractor, at MIT, on $1963,{ }^{10}$ and Christian Mira, discovered in 1978 his chaotic attractor in an iterated quadratic map of the plane creating the theory of critical curves for iterated maps. ${ }^{37,38}$ These discoveries sounded the death knell for our approach based on differential topology.

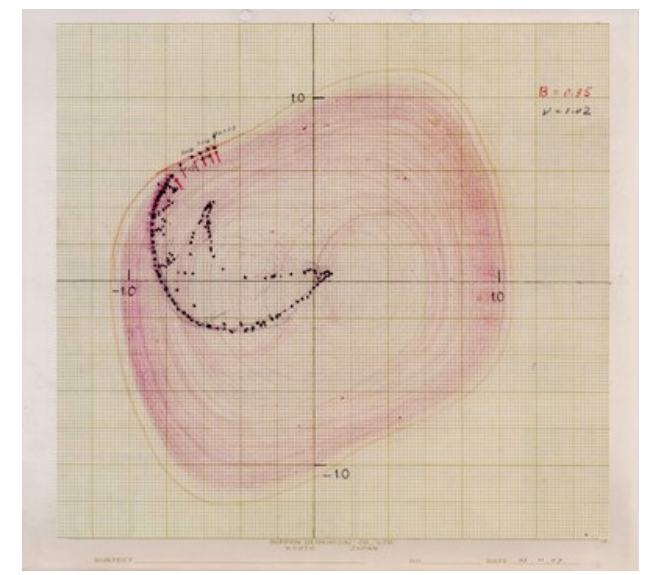

FIG. 2. Yoshisuke Ueda discovered (November 27, 1961) his chaotic attractor in a Poincaré section of the forced Duffing equation (1). Courtesy of Yoshisuke Ueda.

In 1971-73, new people had arrived in my department (UC Santa Cruz), including John Guckenheimer, a recent Ph.D. with Smale, and veterans of our group, Palais and Mike Shub. Palais collaborated in creating a computational program using a digital mainframe and a primitive graphics terminal. We were able to recreate the attractors of Ueda, Lorenz, and Mira, with assistance of a talented group of undergraduates. After a couple years we also studied the Rössler attractor and other new developments. Guckenheimer was also active in computational dynamics at UCSC in the 1970s.

An important meeting, was jointly sponsored in 1977 by the New York Academy of Sciences and the University of Tübingen. My own contribution was the first announcement of my simulation of chaos using digital computer graphics. This work evolved into the graphic introduction for chaos theory written jointly with the artist, Christopher Shaw. ${ }^{39}$
Around 1978, a group of students, primarily Rob Shaw, Doyne Farmer, Norman Packard, and Jim Crutchfield, later known as the Santa Cruz Chaos Cabal (after Gleick's bestseller ${ }^{40}$ ) began a literature seminar and chaos program - the methodology to investigate chaotic attractors with the help of computers, for instance, as synthetized by Lorenz. This resulted in an audacious article in the Scientific American of December, 1986, in which chaos theory reached a wide popular audience for the first time. ${ }^{41}$

\section{BORIS CHIRIKOV - SPUTNIK OF CHAOS BY DIMA SHEPELYANSKY}

Boris Chirikov (1928-2008) was the founder of the physical theory of Hamiltonian chaos and made pioneering contributions to the theory of quantum chaos. ${ }^{42,43}$ In 1959, he invented a simple analytical criterion, now known as the Chirikov criterion, which determines the conditions for the emergence of deterministic chaos in dynamical Hamiltonian systems. ${ }^{44,45}$

There are various research directions launched by Boris Chirikov in the field of chaos. They include chaotic dynamics of particles in plasma magnetic traps and accelerators, chaos border for the Fermi acceleration model, emergence of chaos in various Hamiltonian systems, ${ }^{46,47}$ quantum chaos, ${ }^{48,49}$ in dissipative dynamical systems, ${ }^{50,51}$ and many others.

I joined Chirikov's group at the Institute of Nuclear Physics (INP) in September 1976, at the beginning of my fourth year at the Novosibirsk State University. As many other students, I knew Chirikov from the course of Electrodynamics given by him and Igor Meshkov at our second year. But my choice was also significantly influenced by a recommendation of George Zaslavsky, who had worked with Chirikov and gave outstanding recommendations for his research.

Chirikov was the head of a theory group composed of about ten people working on nonlinear dynamics and stochasticity (now we say chaos); it included essentially Felix Izrailev, Vitaly Vecheslavov and Lida Hailo, who worked as a programmer.

I remember Chirikov's office in 1976-1978. The main focus of the room was a teletype terminal directly connected to a computer BESM-6 at the Computer Center of Siberian Division of Russian Academy of Sciences, located at about $1 \mathrm{~km}$ distance down along Prospect Nauka. This was the most powerful soviet computer at that time. From the terminal it was possible to submit short runs on BESM-6, and even to work in interactive mode. Chirikov defined the main scientific group aim as the investigation of fundamental laws of chaos and foundations of statistical mechanics for classical and quantum systems.

In 1977, the now famous quantum kicked rotator model was invented. The model is the quantized version of the classical standard map, now known as the Chirikov standard map. ${ }^{46,47}$ It has the form

$$
\left\{\begin{array}{l}
\bar{p}=p+K \sin x \\
\bar{x}=x+\bar{p}
\end{array}\right.
$$


where bars mark the new values of conjugated variables of momentum $p$ and coordinate $x, K$ is dimensionless parameter characterizing the kick strength. An example of the Poincaré phase space is shown in Fig. 3. ${ }^{52}$
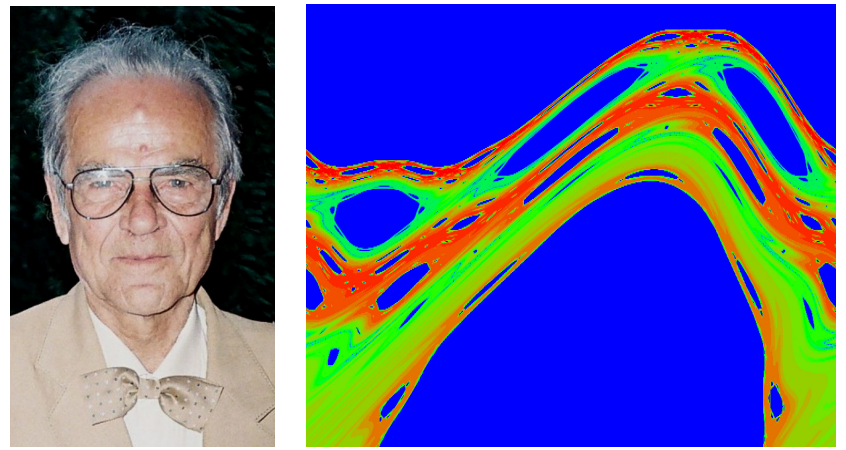

FIG. 3. Left: Boris Chirikov, Toulouse, June 6, 1998. Photo by D. L. Shepelyansky. Right: Amplitude of the eigenstate of the Ulam approximate of Perron-Frobenius operator of the Chirikov standard map at $K=0.971635406$; amplitude is proportional to color with maximum for red and zero for blue; upper part of phase plane is shown for the range $0<\frac{x}{2 \pi} \leq 1,0<\frac{p}{2 \pi} \leq 0.5$. From Frahm et al, 2010 .

Back in the late spring of 1977, Chirikov suggested that I work on the kicked rotator model, starting from the improvements of the computer code. Following his suggestions, I achieved a significant reduction of the CPU time, and I am still proud that the improved figures we obtained, were used in the Russian version of the kicked rotator paper published as INP preprint in $1978 .^{53}$

At those times even chaotic dynamics in nonlinear classical systems was a rather new and unusual subject for the world scientific community. For example, there wasn't any specialized journal in this field, and often it wasn't easy to explain to an editor how it happens that, in spite of Laplace determinism, simple equations produce chaotic unpredictable behaviour. Quite often, editors blamed errors of numerical simulations, and rejected papers on chaos. The world wide circulation of research results was initiated by Joe Ford (Georgia Tech), who, every week, patiently collected the abstracts of new preprints on chaos and nonlinearity, with young collaborator Franco Vivaldi, and send them to colleagues and friends. Chirikov knew Joe Ford from their first meeting in Kiev in 1966, where Ford came as a tourist with a group of school pupils to visit the USSR. Finally, the first specialized nonlinear journal, Physica D, was created in 1980. During many years Ford and Chirikov worked in the editorial board of this journal.

\section{MY DANCE WITH CHAOS BY OTTO RÖSSLER}

The first skill that I developed and which was significant for my contribution to chaos was related to my interest in telephones. It gradually led to repairing and then building radios and radio emitters. I got my radio amateur's licence DL9KF when I was 17 . After my medical studies, I got a first scientific position at the Max Planck Institute for Behavioural Physiology in Biocybernetics at Seewiesen. During that year, I developed a big friendship in very long discussions with Konrad Lorenz. I then spent one year as an intern at the University of Marburg partly under the supervision of Reimara Waible who became my wife, one year later.

I hereafter obtained a one-year position for working with Robert Rosen. In the continuation of Nicholas Rashevsky, the pioneer of mathematical biology, ${ }^{54-56}$ Rosen developed a bridge between dynamical systems theory and biology. ${ }^{57}$ Bob and I had an immediate resonance. From his book I discovered Andronov, Khaikin and Vitt's textbook ${ }^{58}$ which later led me to building a three-variable chemical multivibrator. ${ }^{59}$ Bob's book made me firm in dynamical systems thinking.

A few years before, I had met Friedrich-Franz Seelig. He offered me to join his new group at the University of Tübingen. In the early 60s, Seelig had done his diploma work with Hans Kuhn and Fritz-Peter Schäfer to build an analog computer consisting of a network of electrical oscillators, connected to capacitors to solve the two-dimensional Schrödinger equation. ${ }^{60}$ This system was triggered by means of a radio frequency generator. Sharing an interest for the origin of life, in differential equations and electronics (computers), Kuhn, Seelig and myself we agreed that nonlinear systems like my evolutionary soup and electronic systems were virtually isomorphic. This triggered a cooperation project between Seelig and me to look for reaction-kinetic analogs to electronic circuits. I therefore joined Seelig in 1970. Seelig bought an analog computer — a Dornier DO 240 - equipped with potentiometers, a digital clock and two function generators...

I was free in my research and, started to study few-variable systems. Chaos theory is fun. With three variables, recurrent motions can fall into a dance that is beautiful and nonrepeating and surprising at every round. This dance is chaos. Art Winfree stimulated and paved my way into the fascinating topic of chaos theory. In 1972, Art Winfree had invited me for a talk on chemical automata at Purdue University. ${ }^{59,61} \mathrm{He}$ showed me his later well known beautiful experiments with the Zhabotinsky reaction. ${ }^{62,63}$ We started to exchange letters about interpreting chemical reactions in terms of dynamical system theory. In 1975, we met again at a Chronobiology Meeting held in Vienna where I gave a talk on biological clocks. Art found my talk a little bit boring and asked me whether I could do something more interesting in the context of my liquid automata? ${ }^{64}$ I told him that I was thinking about a three-variable limit cycle that looks like a knot and hence cannot be flattened into a planar circle-like thing for being irreducibly three-dimensional. He replied that this sounded to him like chaos. He told me that he had just attended a conference in Aspen, Colorado, on "chaos" and that he had collected all the papers written on the subject and he would send me a folder with them. Four weeks later I received a big folder with all the papers, most still in preprint form.

The folder included Lorenz's paper of $1963^{10}$ and more recent ones like those by Jim Yorke, ${ }^{2}$ Bob May and George Oster. Art wrote me explicitly that I should do him the favor of finding a chemical version of the Lorenz attractor. ${ }^{65}$ Of course, I didn't succeed. I came up with the idea of a rope 
wrapped about my nose several times before falling down and then coming back up again in a loop. This mental picture was the origin. Next came a characterless turning of the knobs on the analogue computer. Simplifying and reducing the system then was sufficient to arrive at the desired attractor. ${ }^{3}$ I finally got the equations ${ }^{5}$

$$
\left\{\begin{aligned}
\dot{x} & =-y-0.95 z \\
\dot{y} & =x+0.15 y \\
\varepsilon \dot{z} & =\left(1-z^{2}\right)(z-1+x)-\delta z
\end{aligned}\right.
$$

Describing sharp transitions at the two thresholds [Fig. 4(a)] where suddenly things switch from one two-dimensional plane to the other, is a bit demanding numerically. After simplifying the equation as much as possible by trial and error, the "miracle" happened that the two formerly overlaid simple linear two-dimensional flows gave rise to a new everywhere smooth three-dimensional flow ${ }^{3}$

$$
\left\{\begin{array}{l}
\dot{x}=-y-z \\
\dot{y}=x+a y \\
\dot{z}=b+z(x-c)
\end{array}\right.
$$

It is not actually the really simplest one, by the way, since I could find later the still simpler one ${ }^{66}$

$$
\left\{\begin{array}{l}
\dot{x}=-y-z \\
\dot{y}=x \\
\dot{z}=b\left(1-y^{2}\right)-c z
\end{array}\right.
$$

I should add here that the stimulation I had obtained from Ralph Abraham and his school — the "Santa-Cruz kids" was crucial. Norman Packard, Rob Shaw, Jim Crutchfield and Doyne Farmer then jointly coined the name for the attractor. ${ }^{67}$ (a) System (3)

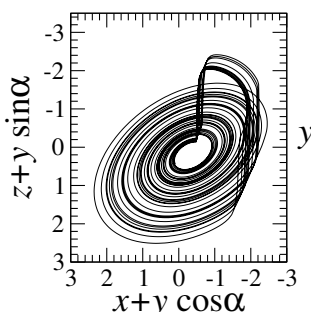

(b) System (4)

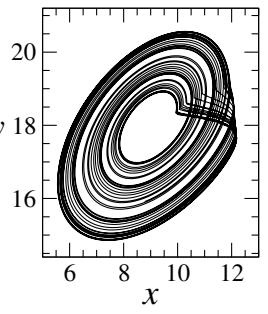

(c) System (5)

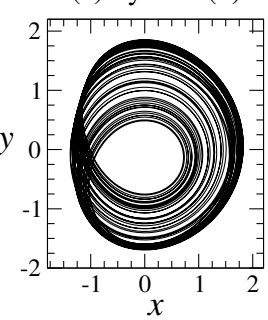

FIG. 4. The chaotic attractor obtained after some simplifications of the original equations. Parameter values for system (3): $\delta=\varepsilon=$ 0.03. For system (4): $a=0.2, b=0.2, c=5.7$. For system (5) $b=0.275$ and $c=0.2$ and initial conditions are $x_{0}=1, y_{0}=-1.4$ and $z_{0}=-0.4$.

The movie with the "sound of chaos" (named after Simon and Garfinkel's "Sound of Silence") was obtained on the analog computer jointly with Reimara. ${ }^{68}$ The sound produced by this simulational reality proved to be familiar to the ear. So chaos is something that is very close to everyday life.

\section{HOW I BECAME INVOLVED IN CHAOS BY PHIL HOLMES}

In 1973 I was finishing a $\mathrm{PhD}$ thesis on noise transmission in structures at Southampton University (U.K.), when I noticed that a course on differential topology and its applications to dynamical systems would be taught by David Chillingworth in the Mathematics Department. This introduced me to René Thom's catastrophe theory and to many analytical tools that I had not known before. While auditing the course, I met David Rand, who was also completing his thesis and who wanted to move towards applied mathematics. We began working together and found an interesting mistake in the interpretation of the amplitude response function for periodic solutions in a preprint of Christopher Zeeman's on Duffing's equation with a stiffening spring $(\alpha>0)$ :

$$
\ddot{x}+2 \zeta \dot{x}+k\left(x+\alpha x^{3}\right)=f \cos (\omega t) .
$$

The mistake involved a misinterpretation of the cusp catastrophe which occurs as a limiting case when 2 curves of saddlenode bifurcations collide in the $(f, \omega)$ parameter space. Zeeman's paper finally appeared in $1976 .{ }^{69}$ We corrected the mistake and sent our preprint to Christopher. He encouraged us to submit and publish, and in due course our paper came out, ${ }^{70}$ followed by further papers on van der Pol's equation ${ }^{71}$ and on a combined Duffing-van der Pol oscillator. ${ }^{72}$ We did not learn of Yoshisuke Ueda's analog simulations of chaos in a similar equation with a cubic damping term until I moved to Cornell University in 1977. Shortly thereafter, Ueda visited Cornell and described some of his results.

However, while still in Southampton David Rand and I found two preprints on nonlinear oscillations by Floris Takens and things began to become chaotic. Takens' papers appeared in $1974 .^{73,74}$ I believe that they established fundamental examples for the classification of bifurcations in dynamical systems.

Cartwright had found a curve of homoclinic bifurcations in a two-dimensional averaged van der Pol equation. ${ }^{75}$ David Rand and I were able to relate it to the homoclinic bifurcations studied by Takens, and thus assemble the correct codimension- 2 bifurcation set. ${ }^{71}$ Motivated by Takens' work, I borrowed an analog computer, from the undergraduate laboratory and (physically) programmed it to simulate Duffing's equation in the form

$$
\left\{\begin{array}{l}
\dot{x}=y \\
\dot{y}=x-x^{3}-\delta y+\gamma \cos (\omega t) .
\end{array}\right.
$$

This system has a potential energy function with two wells separated by a peak. With damping $\delta>0$, but without periodic forcing $(\gamma=0)$, almost all solutions approach one or the other of the two stable equilibria $(x, y)=( \pm 1,0)$, except for the stable manifold of the saddle point $(x, y)=(0,0)$. More significantly for chaos, when damping $\delta=0$, there are two homoclinic orbits beginning and ending at the saddle point. I had read and understood relevant parts of Smale's paper ${ }^{20}$ and realized that this implied that, with sufficiently large periodic forcing $\gamma \neq 0$, the Poincaré map of equation (7) has 
infinitely-many transverse homoclinic points, and therefore contains Smale horseshoes (see Fig. 5 for an example).

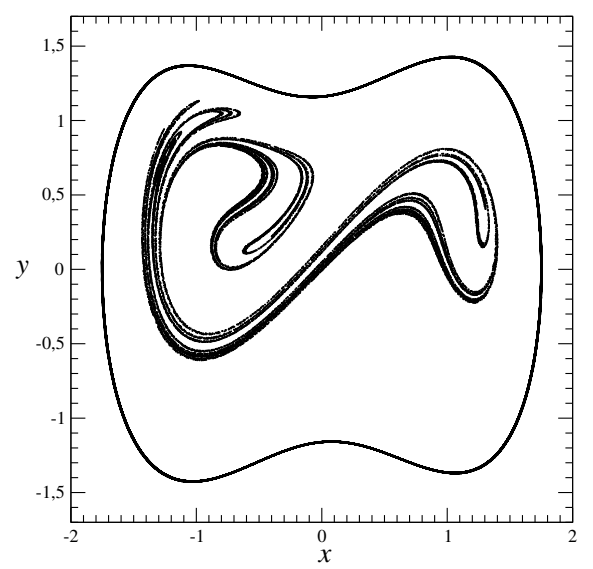

FIG. 5. Coexistence of a chaotic attractor and a large stable period1 limit cycle. Parameter values: $\delta=0.15, \gamma=0.30$, and $\omega=1$. Initial conditions: $x_{0}=1$ for the chaotic solution and $x_{0}=1.8$ for the period-1 limit cycle, and $y_{0}=0$.

Two conferences sponsored by the New York Academy of Sciences in 1977 and 1979 focused on bifurcation theory and nonlinear dynamics, ${ }^{76,77}$ and did much to stimulate the field by bringing diverse researchers together. I chaired a related conference in 1979 which both engineers and mathematicians attended. ${ }^{78}$ Since I had trained as an engineer and at that time was still trying to become an applied mathematician, this meeting was important for my future.

Throughout my early work and collaborations I was most excited by the combination of computer simulations, rigorous mathematical theorems, and physical experiments that, together, could create new models of dynamical processes. The classification of bifurcations played a key role in this.

\section{HOW CHAOS SHAPED MY ACADEMIC LIFE BY RENÉ LOZI}

Strangely enough, although I was very interested in the first examples of chaotic attractors from the end of the 1970s, I never paid attention too much to the Rössler attractor. However, the qualitative procedure of this method was strongly inspiring me during several years, allowing to propose a geometric model of slow-fast Lorenz-like attractor ${ }^{79}$ and the Alpazur oscillator with Hiroshi Kawakami. ${ }^{80,81}$ Moreover, I was interested in his researches on hyperchaos and his prototypic models, ${ }^{82-84}$ map (although non-continuous) three years later. $^{85}$

I started my studies at the University of Nice in October 1967 , in mathematics and physics. I had been taught that there was a list of ODEs written by Bernoulli, Lagrange, Clairaut, Riccati, etc. and a list of solving methods. No physical sense, in fact no meaning at all, was attached to these academic exercises. No numerical method was taught. Moreover, between professors, there was a strict separation between "pure mathematician" and the few "applied mathematician" who were able to use a computer. At the university I took my first programming course about FORTRAN IV in 1968, using punched cards. I discovered with fascination the methods of numerical integration of ordinary differential equations (ODEs). During 1970-71, I was following my bachelor's degree under the supervision of Professor Martin Zerner (1932-2017). Martin was the first guy, who was able to use a computer that I ever met.

During his lectures, my mind knew a breakthrough that changed the paradigm: the set of all the equations I was taught, were of zero measure in the set of all ones existing. No closed formula of solution can be found for most of them. Only numerical methods were able to provide approximate solution. Of course, in this scope, computer was essential. Moreover, ODEs were useful to model physical, chemical or even biological situations. This new paradigm has guided my research career throughout my whole life.

While preparing my Ph.D., 86 the name "bifurcation" was largely unknown in the communities of mathematical and numerical analysis in France. Of course, the term bifurcation was introduced 90 years before by Henri Poincaré ${ }^{87}$ but we must consider that the decade 1960-70 was the golden age of the Bourbaki group, ${ }^{88}$ whose philosophy was drastically opposed to Poincaré's way of thinking. Moreover, Jean Alexandre Dieudonné, one of the founders of the Bourbaki group, arrived at Nice in 1964. He was the most prominent professor from the department of mathematics. Poincaré's works were therefore not at all in my mind.

With Gérard Iooss ${ }^{89}$ I worked on the famous dynamo problem explaining the origin of the magnetic earth field. ${ }^{90} \mathrm{We}$ both attended to a conference in Roma (1977). The opening talk was given by David Ruelle. ${ }^{91}$ In his talk, Ruelle conjectured that, for the Hénon attractor, the theoretical entropy should be equal to the characteristic exponent. This is how I discovered the first example of chaotic and strange attractor [Fig. 6(a)]. ${ }^{92}$ At that time, the term "strange" was used, referring to Ruelle and Takens' paper. Today, we would use "chaotic" rather than strange which now refers to the fractal properties of the invariant set. Nevertheless, this is rather the fractal properties of this attractor which were highlighted by Michel Hénon and astonished the research community. Hénon who explored numerically the Lorenz map using the IBM7040, found difficult to highlight its inner nature due to its very strong dissipativity. Hénon built the metaphoric model

$$
\left\{\begin{array}{l}
x_{n+1}=1-a x_{n}^{2}+y_{n} \\
y_{n+1}=b x_{n}
\end{array}\right.
$$

parameter $b$. With $b=0.3$, the contraction in one iteration is mild enough that the sheaves of the attractors are visible [Fig. 6(a)].

Beyond bifurcation problems, my main interest was focused to discretization problems and the finite element method in which nonlinear functions are approximated by piecewise linear ones. During the Roma conference, I tried to apply the spirit of the method of finite element to the Hénon attractor. Back to Nice on June 15 in the morning, I eventually decided to change the square function of the Hénon attractor, which is $\mathrm{U}$ shaped, into the absolute value function, which has a $\mathrm{V}$ 


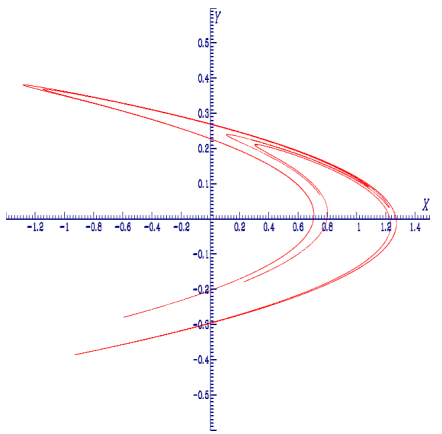

(a)

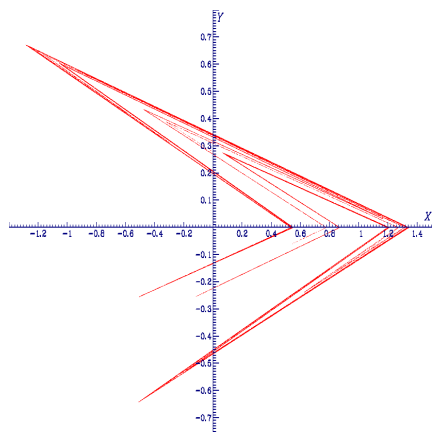

(b)
FIG. 6. Some simple mappings: (a) The Hénon attractor and (b) The Lozi attractor.

shape, implying folding property. I tested this modification, on my small desktop computer HP 9820. I shifted the parameter value $a$ from 1.4 to 1.7 and $b$ from 0.3 to 0.5 (why? I do not remember!) and plotted what is known today as the "Lozi map" [Fig. 6(b)]. ${ }^{93}$ Iooss and Chenciner encouraged me later to publish the formula ${ }^{94}$

$$
\left\{\begin{array}{l}
x_{n+1}=1-a\left|x_{n}\right|+y_{n} \\
y_{n+1}=b x_{n}
\end{array}\right.
$$

This was for me the very beginning of my career in chaotic dynamical systems.

I was convinced that few weeks would be enough to explain and give a proof of the structure of a so simple attractor, but I failed. In the next two years I attended a workshop on iteration theory at La Garde-Freinet (1979) where Michal Misiurewicz, after some questions at the end of my talk, jumped on the stage. On the blackboard he gave some clues of his forthcoming results presented at the famous New-York conference, seven months later ${ }^{95}$ where I am proud to have shook the hand of Edward Lorenz. There I listened with a mix of anxiety and curiosity the first proof by Misiurewicz for the existence of a chaotic attractor for the map I discovered two and half years before. ${ }^{95}$ I was interested in the session devoted to turbulence due to the concept of strange attractor developed by Ruelle and Takens. ${ }^{96}$ The talk by Vidal ${ }^{97}$ on the BelousovZhabotinsky reaction was of a so great interest for me. Of course, the talk by Misiurewicz ${ }^{95}$ was a kind of ecstasy for the young researcher that I was.

\section{MY ROAD TO CHAOS BY LEON GLASS}

For my Ph.D. at the University of Chicago, I studied dynamics of molecules in liquid argon. For postdoctoral studies, I was interested in going back to my original fascination with medicine and psychology. I received a postdoctoral fellowship to study the brain at the newly formed Department of Machine Intelligence and Perception at the University of Edinburgh in 1968.

I returned to Chicago to a Postdoc. Jack Cowan had hired two remarkable young scientists, Art Winfree and Stuart Kauffman for their first faculty positions. Although my initial plan was to continue working on vision, I became intrigued by Kauffman's studies. Kauffman had constructed random Boolean switching networks and found that for networks in which each element only had a couple of inputs, the dynamics was amazingly orderly. ${ }^{98}$ I rejected the Kauffman's notion of discrete states and discrete times, but embedded the switching network logic in differential equations. ${ }^{99,100}$ This was really my first research that involved nonlinear dynamics. I learned about some of the basic notions including bifurcation and stability theory - topics that were not considered appropriate to include in graduate physical science programs at the time. This was immediately before the explosion of interest in chaos.

Michael Mackey, who had training in Biophysics and Mathematics, was a young faculty member at McGill University in Montreal. I had met Mackey at Gordon Conferences in Theoretical Biology in the early 1970s, and I was delighted when the opportunity came to apply to McGill. I moved to Montreal in March 1975 and a few months later went out west to spend a month at the Aspen Center for Theoretical Physics. A talk by Stephen Smale, about the period-doubling route to chaos was intriguing. Mitchell Feigenbaum was also there and he attributed that meeting also to piquing his interest in chaos. ${ }^{101}$

When I got back to Montreal, I was excited to discuss chaos with Mackey. I asked him if physiological systems could display chaos. He said he did not know. We decided to write a team grant application on the theme "Oscillation and Chaos in Physiological Systems." We certainly proposed to study both difference and delay differential equation models for two physiological systems: Mackey would look at hematopoiesis and I would look at respiration. In one of the models for hematopoiesis there were several novel features. There was just one variable - the blood cell concentration. Instead of just using negative feedback, the equation

$$
\dot{x}=\beta \frac{x_{\tau}}{1+x_{\tau}^{n}}-\gamma x \quad \gamma, \beta, n>0
$$

had a non-monotonic feedback term. And since it took some time to produce red blood cells once the signal was received, the production incorporated a time delay term. We searched for chaos in the model. Most exciting was the day when Mackey and I both sat in front of a primitive computer screen and watched the trajectory. Since there was only one variable, we plotted two coordinates, the current value and the value in the past. To the best of my knowledge, this was the first use of time delay embedding to examine complex dynamics and bifurcations. We tweaked parameters and eventually found what we were seeking. ${ }^{102,103}$ It was chaotic (Fig. 7), and the route to chaos seemed similar to what had been observed in simple quadratic maps. ${ }^{2,104}$

General interest in chaos had been piqued by Robert May's 1976 review. ${ }^{105}$ We submitted our findings on chaos in simple mathematical models of physiological systems to Science, and were delighted when it appeared. ${ }^{102}$ We emphasized the concept that diseases could be characterized by abnormal dynamics that might be associated with bifurcations in nonlinear equations. In November 1977, Okan Gurel and Otto Rössler organized a meeting on Bifurcation Theory and 


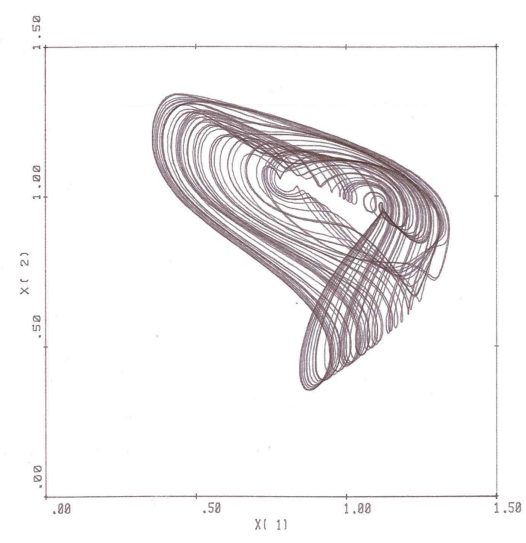

FIG. 7. Time delay embedding of a chaotic time series from the model for blood cell production using the time delay equation (10) that we proposed in $1977 .{ }^{102}$ The trace is similar to the time delay embeddings presented at the New York 1977 conference organized by Gurel and Rössler. Image from the original tracings made in 1976 or 1977 where $x(2)$ is the delayed variable and $x(1)$ is the current variable. $^{103}$

Applications. ${ }^{106}$ I was invited to speak, and ran through a sequence showing the various dynamics in the chaotic time delay equation as a parameter changes using the time delay embedding. ${ }^{103}$

There were many people at the 1977 meeting. One was Robert Shaw, spending significant time to develop a way to beat roulette by entering data from the roulette wheel into a computer program in a shoe. ${ }^{107}$ The Dynamical Systems Collective wrote an influential paper in 1980 showing how you could get a two dimensional portrait of a time series by plotting the value of one variable on one axis and its derivative, or as suggested in a footnote its value at an earlier time, on the other axis. ${ }^{108}$ One member of the group, Farmer, went on to study the time delay equation modeling blood cell dynamics for his doctorate, referring to it as the Mackey-Glass equation! ${ }^{109}$ Another person at the meeting was David Ruelle. Ruelle suggested to me that I could look at the return map to a cross section on the time delay embedding. He correctly thought that it would be parabolic. As far as I know the only published return plot for this equation appeared in a Scholarpedia review article that Mackey and I presented many years later, when we finally took Ruelle's suggestion. ${ }^{110}$

\section{FIRST CHAOTIC STEPS BY ARKADY PIKOVSKY}

As a second-year physics student at the Department of Radiophysics at the University of Gorky, I had to decide the direction of my studies. In early 1974, I approached Michael Rabinovich, that time reader at the Theory of Oscillations chair, and asked if I can do specialization under his supervision, and he agreed. He gave me some review articles ${ }^{111,112}$ to read. I understood very little of them. Nevertheless, when he formulated a first research project - deriving a kinetic equation for modes for the Rayleigh-Bénard convection problem, I started to read books and articles, and almost the whole third year in the University struggled with nonlinear equations for convection. So I read what was relevant to this field in the literature, and at the beginning of 1975 , read a paper by J. McLaughlin and P. Martin. ${ }^{113}$ They wrote about a strange attractor in convection, and I understood nothing.

This paper contained only the 13th citation of the famous 1963 Lorenz paper ${ }^{10}$ and only the 11 th citation of the equally important 1971 paper by Ruelle and Takens, ${ }^{1}$ and it was the first publication that cited both. McLaughlin and Martin matched Lorenz's nonperiodic flow with the strange attractor concept. As a matter of fact, stochastic dynamics (that is how deterministic chaos was called in the Russian literature that days) was a known concept due to works of Boris Chirikov and his group, ${ }^{114}$ but a general belief was that conservative Hamiltonian chaos does not survive dissipation, and in dissipative systems only limit cycles can be stable (robust) attractors. Lorenz's model and the theoretical concept of Ruelle and Takens demonstrated that dissipative chaos could be permanent, and more and more examples of it appeared in 1975. To us, these novel ideas came, not in a direct way of reading the McLaughlin-Martin paper, but through the mathematical group on dynamical system theory around Ya Sinai in Moscow. ${ }^{115,116}$ M. Rabinovich, together with Svetlana Vyshkind, studied low-dimensional models of nonlinearly coupled modes and observed irregular dynamics there. ${ }^{117}$ He met Ya Sinai and from him got to know about the concept of strange attractors. M. Rabinovich returned from Moscow very enthusiastic about this, and I was eager to learn more in this direction (at this point I also realized that I had already read about this in McLaughlin-Martin paper). M. Rabinovich brought from Moscow several preprints and lecture notes that Sinai gave him (I remember it was a text by O. Lanford III among them) which I tried to read. But this was rather hard for a non-mathematician, with some objects like "Axiom A" that I could not identify. Thus I took a step back and started from more basic texts like translated to Russian Smale's review ${ }^{20}$ and lectures by A. Katok and others at Russian mathematical schools.

Around the middle of 1976, M. Rabinovich first realized that there is a close analog of the Lorenz system in the realm of coupled oscillators (or oscillatory modes). The linear terms in the Lorenz model can be interpreted as a combination of dissipation and parametric excitation, while the nonlinear terms correspond to a "classical" three-mode resonant interaction. This model is known as the Rabinovich system ${ }^{118}$

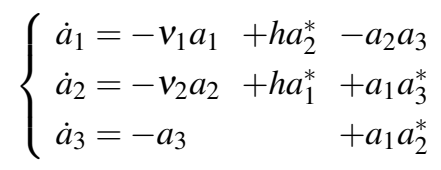

where the system is written in three columns, with dissipative, excitation, and nonlinear coupling terms, correspondingly. Remarkably, the complex solutions of the Rabinovich system appeared to lie on the real-valued three-dimensional manifold [Fig. 8(a)], what made the analogy with the Lorenz system nearly perfect.

The second idea came after a paper by Rössler. ${ }^{3}$ There he argued that in a three-dimensional slow-fast system, with a 
two-dimensional S-shaped slow manifold, one can reduce the dynamics to a one-dimensional non-invertible map and thus get chaos. Slow-fast systems were a popular object at the Theory of Oscillations chair, in the context of electronic circuit dynamics. M. Rabinovich decided to construct a chaotic electronic generator with slow-fast dynamics.

Working on these two problems was an exciting time for me. The computations' results had to be put to the graphs (on a graph paper) by hands. we used an analog computer with a plotter. One could easily arrange a simple set of equations on this analog computer, but accuracy was miserable. So one just adjusted parameters (through rotation of a potentiometer) to obtain a beautiful plot. Moreover, while plotting a long trajectory, parameters could deviate, and it suddenly exploded. In Fig. 8 I present analog computer phase portraits of the Rabinovich system and of the slow-fast dynamics in an electronic circuit from papers. ${ }^{118,119}$

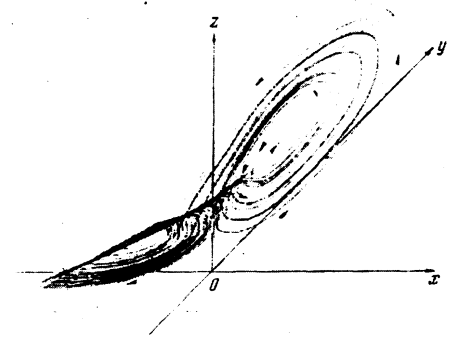

(a) Rabinovich system

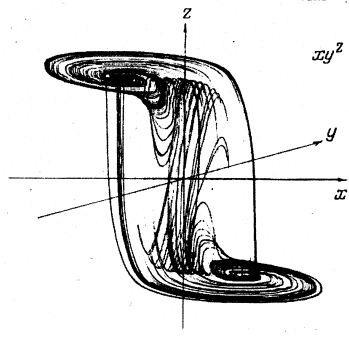

(b) Chaotic generator

FIG. 8. Phase portraits of two strange attractors created on an analog computer produced by (a) the Rabinovich system (11) and (b) the electronic chaotic generator. Reproduced with permission (a) from Zhu. Eksp. Teor. Fiz., 74, 1366 (1978) and (b) from Dok. Akad. Nauk SSSR. 239, 301 (1978). For both, copyright 1978, Russian Academy of Sciences.

\section{TÜBINGEN BLUES BY LARS FOLKE OLSEN}

My own introduction into the field came when I first went to the department of biochemistry, Odense University (now University of Southern Denmark) as a graduate student in 1975 to study bistability and oscillations in a single enzyme reaction known as the peroxidase-oxidase reaction. My supervisor was professor Hans Degn, who had studied this and other oscillating chemical reactions since the early 1960 s. ${ }^{120,121}$ In those days the typical project for a biochemistry student was to purify a new enzyme (or a known enzyme in a new organism), establish an assay to measure its activity and finally determine its $\mathrm{K}_{M}$ and turnover. However, since my high-school days I had a crush for mathematics and physics.

In the fall of 1975 Hans Degn urged me to attend a meeting on "Rhythmic Functions in Biological Systems" in Vienna (September 8-12). The meeting was mostly on circadian rhythms and I did not know any of the participants and also had nothing to contribute. However, I had the pleasure of meeting two scientists who have had a great influence on my later career. One was Arthur Winfree and the other was
Otto E. Rössler. Back in Odense Degn informed me that some unspent money could be used for a month visit to a lab of my own choice. I asked Otto, if he would be willing to have me around for a few weeks.

When I arrived Otto had just submitted his first paper on chaos in a (bio)chemical system. ${ }^{3}$ My plan was to make a model of the PO reaction that could unify its ability to show both bistability and oscillatory behavior based on some enzyme-kinetic measurements done in the lab. Otto helped me with the model and in fact we did get it to work. The equations are

$$
\left\{\begin{array}{l}
\dot{a}=K\left(a_{0}-a\right)-\frac{V\left(a+\kappa a^{2}\right) b}{\lambda b+a+\mu a^{2}} \\
\dot{b}=\sigma-2 \frac{V\left(a+\kappa a^{2}\right) b}{\lambda b+a+\mu a^{2}}
\end{array}\right.
$$

where $a$ represents $\mathrm{O}_{2}$ and $b$ represents NADH. $K$ is a constant that determines the rate of diffusion into the reaction mixture and $a_{0}$ represents the $\mathrm{O}_{2}$ concentration at equilibrium. $V, \kappa, \lambda$ and $\mu$ are enzyme kinetic paramaters and $\sigma$ is the inflow rate of NADH. The model showed coexistence of steady state and limit cycle oscillations with an unstable periodic orbit separating the steady state and the limit cycle. It was never published in full, but the experimental data underlying the model appeared in a later publication. ${ }^{122}$ When discussing this model Otto was also telling me about an interesting new kinetic model he had made which could show nonperiodic oscillations, sensitive to initial conditions, which he referred to as chaos.

Following my short stay in Tübingen I started a new series of experiments with an open system where both substrates NADH and $\mathrm{O}_{2}$ were supplied continuously to the reaction mixture containing the enzyme. Much to our surprise, the resulting oscillations were different and far more complex than we had anticipated. We observed mixed-mode oscillations and bursting oscillations, none of which could be explained by my simple two-variable model. Sometimes we also observed non-periodic oscillations as mixtures of small and large-amplitude oscillations in a seemingly random order (Fig. 9). Initially, Degn dismissed these oscillations as artefacts generated by small random fluctuations in the pumping rate of NADH inflow, but they appeared consistently when repeating the experiments with the same experimental settings. I had an idea that these non-periodic oscillations could be chaos, and therefore I wrote a letter to Otto with an extensive description of what I had done with free-hand drawings of the data. Otto's reply was: "Read Lorenz!!"”10

I read Lorenz's fascinating paper ${ }^{10}$ and more recent papers by Robert May ${ }^{104}$ and $\mathrm{Li}$ and Yorke ${ }^{2}$ and suddenly I understood. Following Lorenz's instructions I constructed a return map by plotting each amplitude from our irregular PO oscillations against the preceding amplitude (Fig. 10) and applied the $\mathrm{Li}$ and Yorke theorem. ${ }^{2}$ I showed the plot together with the papers by Lorenz and Li and Yorke to Degn, who immediately changed his opinion on the results. Within a week we had written the manuscript and submitted it as a letter to Nature by the end of October 1976. We also sent copies 


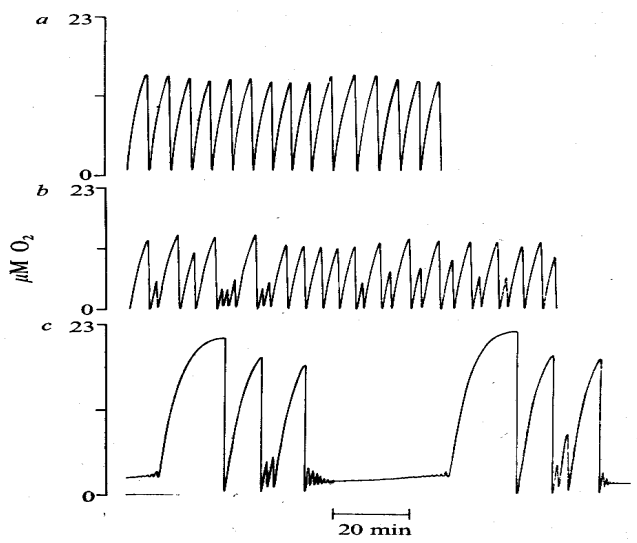

FIG. 9. Simple periodic (a), chaotic (b) and bursting (c) oscillations of $\mathrm{O}_{2}$ in the peroxidase-oxidase reaction. Reproduced with permission from Nature 267, 177 (1977). Copyright 1977, Springer Nature.

of the manuscript to Otto and to Art Winfree, from whom we received very enthusiastic responses. ${ }^{123} \mathrm{~A}$ few months later the paper by Schmitz, Graziani and Hudson on chaos in the Belousov-Zhabotinskii (BZ) reaction appeared. ${ }^{124}$ In 1978 Otto and Klaus Wegmann also published a paper on chaos in the $\mathrm{BZ}$ reaction. ${ }^{125}$ It is important to note that in those days Takens' embedding method ${ }^{126}$ had not yet been published.

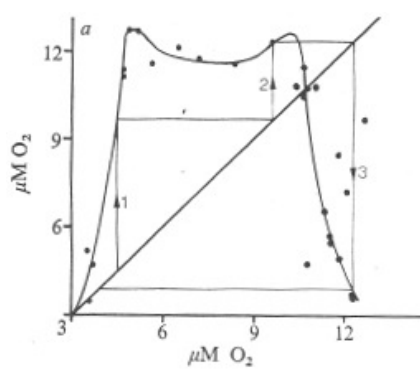

(a) On the amplitude

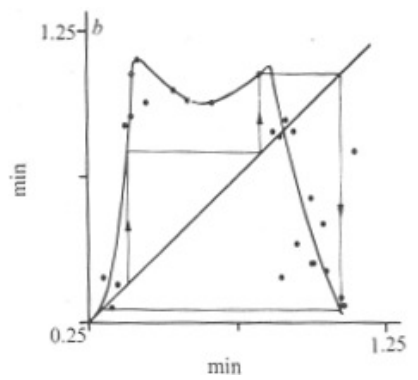

(b) On the time-period
FIG. 10. a) Next-amplitude map of oscillations of $\mathrm{O}_{2}$ from Fig. 9(b); b) same type of plot for the oscillation periods. Trajectories with arrows were drawn to show that the transition function allow the period 3. Reproduced with permission from Nature 267, 177 (1977). Copyright 1977, Springer Nature.

\section{$X$. FROM CHEMICAL CHAOS TO CHAOTIC BRAIN BY ICHIRO TSUDA}

As everyone does, I enthusiastically studied Otto E. Rössler's pioneering works on chaos $5,6,82,84$ in my graduate student days, in the late 1970s. I tried to understand the mathematical structure of Lorenz chaos ${ }^{10}$ in relation to Smale's horseshoe map, ${ }^{20}$ the relationship between Lorenz chaos and a strange attractor ${ }^{1}$ in a sense of mathematical representation of hydrodynamic turbulence, and also the relationship between such chaos and chaos in a sense of LiYorke, ${ }^{2}$ while I kept thinking of "real" chaos observed in the Belousov-Zhabotinsky (BZ) reaction system. ${ }^{124,127-130}$ Otto's contribution $^{131}$ to real chaos in that system with Klaus Wegmann encouraged me to pursue this direction of research.

Here, let me add some more comments about my early research with the late Kazuhisa Tomita, concerning chaos in the $\mathrm{BZ}$ reaction. We noticed early reports of chaotic behaviors in this chemical reaction system. One report was published by Wegmann and Rössler ${ }^{131}$ mentioned above, while the other was by Schmitz, Graziani, and Hudson. ${ }^{124}$ Although they reported "chaotic" behaviors in laboratory experiments, it was not clarified that those behaviors can be characterized by mathematical structure recognized as deterministic chaos or strange attractors. We wanted to show definite evidence for the presence of chaos in the BZ reaction. We thought that finding evidence was easy. The reason was that Otto already showed the presence of chaos in three-dimensional continuous chemical reaction systems even with one quadratic nonlinear term, and further the BZ reaction system should include more than one quadratic nonlinear terms due to molecular collisions of two different chemical substances.
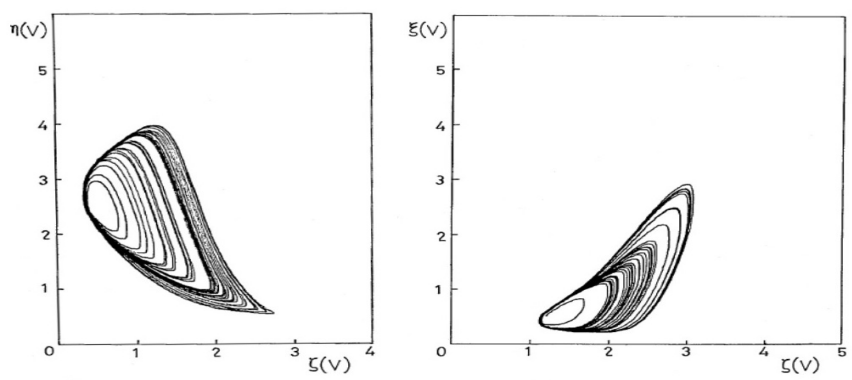

FIG. 11. "Chaotic" orbits yielded by the analog computer at Yoshisuke Ueda's laboratory in 1978 by using the model (13). Parameter values: $p \approx 90, \phi \approx 0.048$, and $m \approx 3.44$. Figures reproduced from the Ichiro Tsuda's Master thesis, Kyoto University, 1979.

For the first time, we made a three-dimensional continuous model for the BZ reaction, based on an original Oregonator proposed by Field and Noyes. ${ }^{132}$ In 1978, Kazuhisa Tomita asked Yoshisuke Ueda to allow us to use analog computers in his laboratory. I finally found "chaotic" behaviors in our model

$$
\left\{\begin{array}{l}
\dot{\xi}=(1-\phi) \xi+\eta-\xi \eta-\xi \zeta \\
\dot{\eta}=-(1+\phi) \eta+\zeta-\xi \eta+m \\
p \dot{\zeta}=\xi-(1+p \phi) \zeta-\xi \zeta
\end{array}\right.
$$

using analog computers (Fig. 11). ${ }^{129}$ Christian Vidal's Bordeaux group found very similar chaotic behaviors to our findings in their laboratory experiment. ${ }^{128}$ We were excited by this experimental finding. Unfortunately, however, I could not find any chaotic behaviors by digital computations with our model. The equations used in analog and digital computations were the same, but the computation results were different: one showed chaotic behaviors and the other showed simply periodic ones. I guessed that the chaotic behaviors found in the analog computer could be a kind of noise-induced chaos caused by the weak stability of limit cycles. Our model does not have either an additional geometric structure produc- 
ing chaos as in the Rössler's system or an additional dynamical rule for changing the bifurcation parameter. One more variable was necessary for yielding deterministic BZ chaos. Therefore, I guessed the findings to be noise-induced chaos. Finally, I gave up making a continuous model for deterministic chaos of the BZ reaction. Instead, I concentrated on finding mathematical structures showing the existence of deterministic chaos in the experimental data. ${ }^{130}$

\section{REMINISCIENCE OF CHAOS BY CELSO GREBOGI}

In mid-seventies, while working on my Ph.D. thesis in thermonuclear fusion, I took a course in the qualitative theory of differential equations with a visiting mathematician. It was a cautious, abstract four-month long course on specific differential equations. As I was about to discover soon after, in that course there was none of the bold, intuitive philosophical generalisations that James Clerk Maxwell, ${ }^{133}$ a physicist, and Henri Poincaré, ${ }^{134,135}$ a mathematician, felt to be justified. Both understood the importance of systems having sensitive dependence on initial data, the kind of dynamics that is vibrant, compelling and exciting.

In 1978-1981, I became a postdoc in Berkeley under Allan Kaufman. During that time, a few markedly important events occurred related to chaos. Still during the Soviet times, Boris Chirikov came from Novosibirsk to visit Kaufman in the Autumn of 1978. He brought and left with us a preprint copy of his seminal work. ${ }^{47}$ With Chirikov preprint on hand, Kaufman organised a discussion group, three hours every Thursday afternoon, initially to study Chirikov's paper, later to go over V. I. Arnold's recently published book. ${ }^{136}$ In the discussion group, there were we - Kaufman's group, his former students, and some mathematicians. The latter ones were really important because we learned from them the fundaments of the theory of dynamical systems and ergodic theory, necessary to embark in this new science. The learning of a new science, chaotic dynamics, supported by both the ergodic theory and the theory of dynamical systems, was the most exciting aspect of the multiple-year discussions.

Motivated by the studying of the Chirikov preprint, Kaufman asked the student Steven Mcdonald to solve the Helmholtz equation in the chaotic Bunimovich stadium. Their seminal work on quantum chaos. ${ }^{137}$ About the same time, Sir Michael Berry, came to Berkeley to deliver the physics colloquium. He spoke about his work on the swimming pool hot spots and on the twinkling of the stars, ${ }^{138}$ perhaps the two most important examples that can be understood by employing the catastrophe theory of Thom. ${ }^{139}$ It was a fascinating talk that stimulated me to read Thom's catastrophe work. We tried to apply the theory to particle and wave propagation but without much success.

In the Autumn of 1981, I moved back to the University of Maryland, where another chapter in chaotic dynamics was about to take place. Upon arriving in Maryland, I delivered a course on symplectic dynamics and Lie transforms at a Navy lab. That invitation came from Robert Cawley, who felt that the theory of dynamical systems was the way of the future.
There I met Louis Pecora. As part of that course, I invited James Yorke to deliver a seminar as a guest. I have never met him before, though I saw him walking on campus around 1977. I was slightly aware of his work while at Kaufman's group. After his talk, we sat on the stairs in front of the building, chatting about his mathematical work on dynamical systems, and about the naming of "chaos".2

That initial conversation with Yorke was the beginning of a two-decade long collaboration, involving the renowned physicist, Edward Ott, resulting in well over one hundred papers on the fundaments of chaotic dynamics in such a collaboration. Our work, grounded on the theory of dynamical systems and ergodic theory, and often argued in terms of point set topology, were developed with the use of mathematical maps and differential equations. The latter, typically the pendulum equation $^{140}$

$$
\ddot{x}+v \dot{x}+\omega^{2} \sin x=f \cos t
$$

was usually employed to argue that the phenomenon we were addressing was not particular to a mathematical framework but it was pervasive in science and technology. In fact, the objective of the research was to establish basic mathematical principles so that researchers could then apply those principles to understand and analyse the systems they were investigating in their own fields.
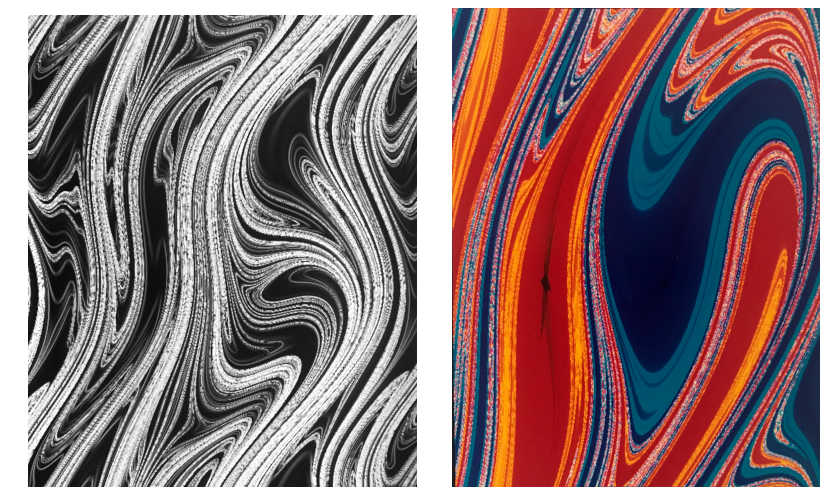

FIG. 12. Fractal basin boundary and the basins of attraction of the forced damped pendulum equation (14). Parameter values: $v=0.1$, $\omega=1$, and $f=2$. Reproduced with permission from Science 238, 632 (1987). Copyright 1987, American Association for the Advancement of Science.

Visualisation was a major component in the early scientific developments of chaotic dynamics. It was essential to be able to draw pictures of attractors, basins of attraction, and other invariant sets on a sheet of paper or project them on a screen. In the late seventies and early eighties, it was difficult, or often not doable, to carry out more intricate calculations in order to help visualisation and understanding, and to validate the theories and predictions. ${ }^{141}$ We hired a technician from NASA to programme and to deal with the idiosyncrasies of computer array. Figure 12, showing the fractal basin boundary and the basins of attraction of a forced damped pendulum equation, is the result of such computations in that computer array. ${ }^{142}$ Our pictures were exhibited at the National Academy of Sciences, in a museum in New York, and were part of a travelling 
exhibition throughout the United States. They were also the covers of a dozen of mathematical, scientific, and technical publications.

\section{HOW I BECAME A NONLINEAR DYNAMICIST BY ULRICH PARLITZ}

In 1978 I started studying physics at the University of Göttingen and in 1982 I was looking for an interesting topic for my diploma thesis. In those days I read popular science books on synergertics, self-organization and evolution theory written by H. Haken, ${ }^{143}$ I. Prigogine \& I. Stengers ${ }^{144}$ and M. Eigen \& R. Winkler ${ }^{145,146}$ and I was fascinated by these new emerging fields, because they addressed very fundamental questions of human live and existence, on how structure comes into being, how units of increasing complexity and functionality arise as a consequence of natural dynamical laws, and in which sense all these processes can be predicted (or not). When talking to fellow students I got the hint that Werner Lauterborn works on nonlinear dynamics and chaos and I should talk to him about a diploma thesis in his group. I did so and soon after he suggested me to investigate the dynamics of a periodically driven Duffing oscillator

$$
\ddot{x}+d \dot{x}+x+x^{3}=f \cos (\omega t) .
$$

Werner was primarily interested in nonlinear resonances he found in his pioneering work on acoustically driven gas (cavitation) bubbles in a liquid in the $1970 \mathrm{~s}^{147}$ and one of my first tasks was to look for such phenomena in the parameter space of the Duffing oscillator. Experimentally it was shown by him and E. Cramer in $1981^{148}$ that bubble dynamics can exhibit period-doubling cascades to chaos, in this context also called acoustic cavitation noise. So searching for chaos was also on my to-do list. It was known that chaotic attractors exist for the Duffing equation with a double-well potential. ${ }^{149}$ For the single well oscillator (15) such theoretical results did (to our knowledge) not exist.

So I started to work, most of the time in the university computer center filling the queue of their main computer, a Sperry UNIVAC 1100/82, and using a VAX-11/780 for interactive exploration of the Duffing oscillator to learn more about its dynamics and how it changes when varying the driving frequency $\omega$ or the driving amplitude $f$. A surprisingly rich, complex but ordered structure of resonances, bifurcations and coexisting periodic and chaotic attractors emerged which fascinated both of us, Werner and me (Fig. 13). ${ }^{150}$ If this nonlinear system, which looks so simple, already produces such a wide variety of dynamic behavior, what would happen with more complex, higher dimensional systems?

This was also the time when I listened for the first time a seminar talk given by Otto Rössler at the University of Göttingen. It was so impressive that I still remember the situation in the seminar room when Otto Rössler showed is slides with the taffy puller to explain the mechanism of stretching and folding underlying chaotic dynamics in state space.

After I had finished the diploma thesis I continued with my Ph.D. studies in Werner's group and delved deeper into

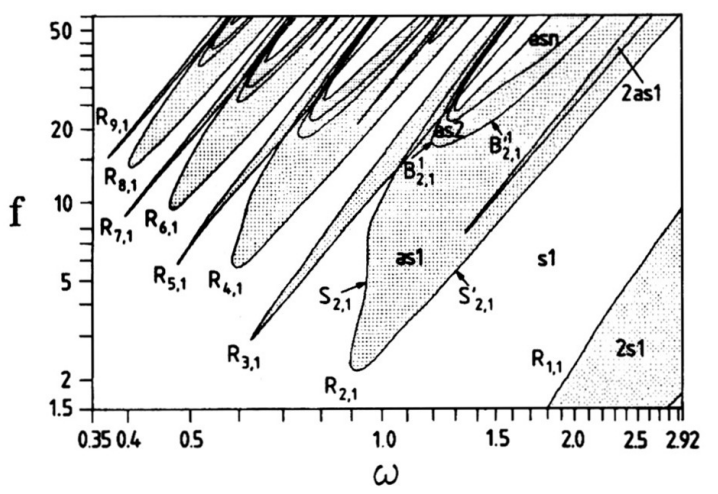

FIG. 13. Parameter space of the Duffing system (15). In the differently dotted domains there are symmetrical period-1 limit cycle (s1), two coexisting symmetry-related period-1 limit cycle (as1), two pairs of asymmetry related period-1 limit cycles (2as1), a pair of period-2 symmetry-related limit cycles (as2) and pairs of period- $n$ symmetryrelated limit cycles (as $n$ ) with $n=4,8,16, \ldots$ Reproduced with permission from Phys. Lett. A. 107, 351 (1985). Copyright 1985, Elsevier.

the dynamics of periodically driven oscillators. How could one characterize and label the nonlinear resonances and bifucation curves seen in the Duffing equation (15), the periodically driven bubbles, ${ }^{147}$ and other nonlinear oscillators? We knew the dynamics of the circle map and the theory of Arnold' tongues but this was applicable only to self-sustained (or selfexcited) systems like the van der Pol oscillator. So the question was, where can we find a second "rotational motion" in addition to the periodic driving in order to compute and analyse their frequency ratio? The solution was to consider the winding of neighbouring trajectories around the periodic orbits and to quantify this motion by torsion numbers. ${ }^{151,152}$ The (average) local torsion frequency $\Omega$ can also be used to define a winding number $w=\Omega / \omega$ that fulfills particular recursive schemes in period-doubling cascades and is also defined for chaotic attractors of this class of systems. This approach was not only applied to the Duffing oscillator, a model for a periodically driven gas bubble, ${ }^{153}$ and many other passive nonlinear oscillators ${ }^{154}$ but also to the periodically forced van der Pol oscillator $^{155}$

$$
\ddot{x}+d\left(x^{2}-1\right) \dot{x}+x=f \cos (\omega t) .
$$

The main motivation for our study of the van der Pol oscillator was, however, the fact that it was known since the seminal analytical work of M.-L. Cartwright and J. E. Littlewood ${ }^{156}$ that this system may exhibit aperiodic oscillations, but we could not find in the literature any numerically computed example of a chaotic attractor for Eq. (16). In fact, it took some detailed numerical simulations until we found a complete period-doubling cascade to chaos. ${ }^{155}$

\section{A KNOTTED ROAD TO CHAOS BY ROBERT GILMORE}

My trajectory as a physicist was strongly perturbed by Fortunato Tito Arecchi. He is a world-class laser physicist who 
visited M.I.T. for a year around 1970. Learning how the operating state changed as the parameters changed was a bifurcation theory problem. ${ }^{157}$ We did a lot of useful work in this field; besides it was fun. At this time a strong connection between the laser physics community and the nonlinear dynamics community was established by Haken. He showed ${ }^{158}$ that there was a deep connection between one of the standard laser physics models in a certain limit and the behavior of fluids as described by the Lorenz equations. ${ }^{10}$ This connection provoked a number of experimental searches for Lorenz-like output behavior of various types of lasers. ${ }^{159,160}$

During this period I encountered a reference to "catastrophe theory'. Somebody pointed me to Thom's book, ${ }^{32}$ not yet translated into English. After reading it I understood nothing, and put this down to my halting French. By perserverence and luck I was directed to Tim Poston, then in the process of writing his book on the subject with Ian Stewart. ${ }^{161}$ Tim gave me copies of several important draft chapters. They were so wellwritten and straightforward that the concepts were easily assimilable. Tim also directed me to a forthcoming work of Erik Christopher Zeeman ${ }^{33}$ which put the subject to work through many imaginative examples - many too imaginative for the staid physics community. The important take-aways from this diversion were: ${ }^{162}$ i) the most visible singularities are the stable nodes but the most important are the unstable saddles because their eigendirections help define basin boundaries; ii) bifurcations on manifolds could have canonical forms; iii) all the important ones were discretely classifiable, and iv) the classification overlapped enormously with the classification of simple Lie groups. This was my introduction to chaos, both of maps and flows, and the Lorenz attractor. ${ }^{10}$

The study of chaos changed dramatically around this time. The enormously powerful tools of renormalization group theory ${ }^{163,164}$ were applied to iterative maps in the late 1970 s on both sides of the Atlantic. ${ }^{165-168}$ These results rapidly lead to several new invariant quantities, such as the scaling ratio $\delta=4.669 \ldots$. Once 'universality' was claimed, a sea change occurred. With the universality claim "It was a very happy and shocking discovery that there were structures in nonlinear systems that were always the same if you looked at them the right way." 40 The community of experimental scientists took this as a challenge, and the race was on. Some of the early experimental tests of the universality prediction are reprinted in the excellent collection by Cvitanović. ${ }^{169}$

One set of experiments was carried out by Arecchi and Jorge R. Tredicce and colleagues. ${ }^{170}$ This experiment confirmed universality within experimental error. Tredicce moved to Drexel University to work with Lorenzo M. Narducci in 1985. I moved to Drexel somewhat earlier (1981). My reasons were in part: to continue working on laser problems with Narducci.

In doing these experiments the Arecchi/Tredicce group had collected a great deal of data, Tredicce wondered how he could understand them. This was an exciting challenge that I eventually turned my complete attention to. The data showed, among other things, multiple coexisting basins of attraction surrounding orbits of various low periods that sometimes came into existence or winked out of existence with a small change of parameter. ${ }^{170-172}$ Experience with catastrophes indicated the presence of saddle-node bifurcations.

The observables were the periodic orbits, and the most important ones were the unstable periodic orbits - again, a lesson from catastrophe theory. We defined the relative rotation rate. ${ }^{173}$ The ensemble of these fractions for any pair of orbits had very restrictive and informative properties. Furthermore, they could be extracted from experimental data and compared with models of the system. In this way we were able to show that the dynamics of the periodically driven laser were those of a suspension of the Smale horseshoe. ${ }^{20}$ Not surprisingly, there was a simple relation between the linking numbers of two orbits and the sum of their relative rotation rates. This method was applied to other periodically driven systems. ${ }^{174}$ Then it was extended to autonomous three dimensional dynamical systems as the Rössler system ${ }^{3,5}$ with the standard parameters.

At this point we became aware of the Birman-Williams theorem. ${ }^{175,176}$ This became a key tool for us. We used it as follows. We could extract a set of low-period unstable orbits from a chaotic attractor and then pairwise compute their 'experimental' linking numbers. Then we could propose a branched manifold that might be the projected limit of the attractor. A following comparison of the experimental linking numbers with those derived from the branched manifold would show either that we 'nailed' the analysis or had to go back to the drawing board. The net result was that we were able to classify the topological structure of chaotic attractors by integers. ${ }^{177}$ We were then able to analyze chaotic time series and search for the integer representation of the dynamics that generated these data. ${ }^{178-180}$
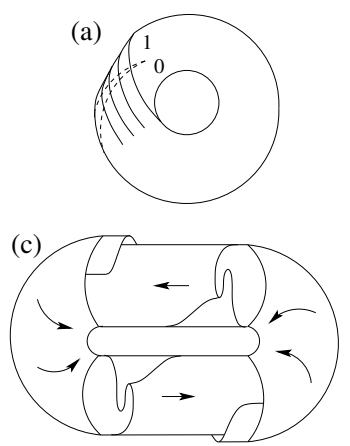

(b)
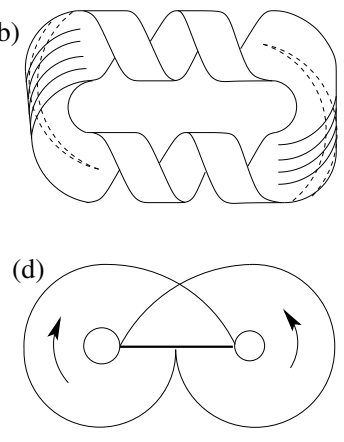

FIG. 14. Branched manifolds describing (a) Rössler, (b) periodically driven Duffing, (c) van der Pol attractors, and (d) Lorenz attractors and for particular parameter values. Redrawn from The topology of chaos, p. 133, (Wiley, 2003).

\section{CHAOS RESEARCH AT THE NAVAL RESEARCH LABORATORY BY LOU PECORA AND TOM CARROLL}

Chaos research at the U.S. Naval Research Laboratory (NRL) was started in the 1980s by Lou Pecora and Tom Carroll, physicists in the former Metals Physics Branch of the Materials Science and Technology Division (MSTD) and by Ira Schwartz originally in Optical Sciences division of NRL. 
Lou wanted to study nonlinear phenomena, especially chaotic motion, in a real system, one that would have interest to the Navy. The material yttrium iron garnet (YIG), a ferrimagnetic compound used in many radiofrequency applications, appeared to be a good candidate material for experimentation. Work by Prof. Carson Jeffries at the University of California, Berkeley, showed that the magnetic spin waves in YIG were a nonlinear dynamical system and could display chaotic behavior. ${ }^{181}$ Lou got Fred Rachford, also in our Metals Physics Branch, interested in the experiments.

Because Rachford had the experiment ready to go, Tom Carroll was able to jump right in and start taking data on chaos in spin wave interactions in YIG spheres in 1987. The results of the experiment did at first appear "chaotic," but not in the sense Tom was looking for. He did notice some strange transients, where the output from the experiment would at first appear chaotic, but then suddenly become periodic. Lou and Tom traveled to the University of Maryland to consult with Celso Grebogi and Ed Ott, who showed them that these transients were something that Grebogi, Ott, and Jim Yorke had actually predicted theoretically. ${ }^{182}$ Ott explained the scaling of the transient times with applied power. Our data showed that the average length of these chaotic transients as micro-wave power was increased fit the predicted theoretical power law. By March 1987, Rachford and Tom had accumulated enough data to allow Lou to present the results at the American Physical Society that month. ${ }^{183,184}$

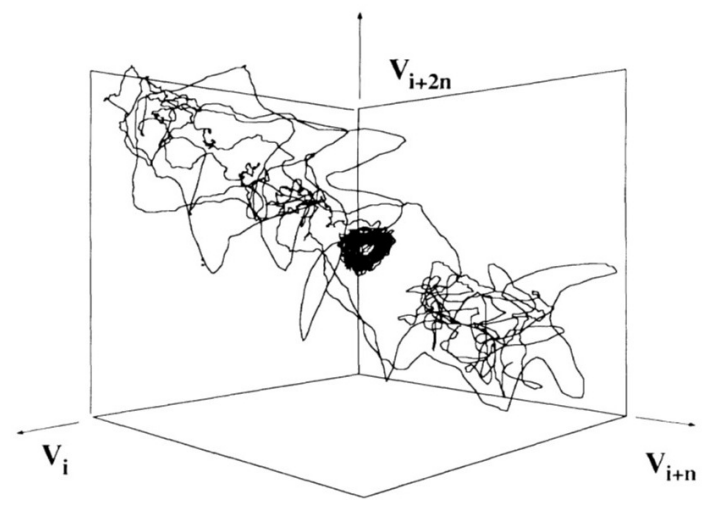

FIG. 15. Trajectory of a chaotic transient ending in a periodic attractor. Reproduced with permission from Phys. Rev. Lett. 59, 2891 (1987). Copyright 1987, American Physical Society.

Tom and Lou talked about synchronizing chaotic systems, but they couldn't come up with any clear way to do that. In January 1988, Lou came home tired from the trip and after dealing with his young daughter late at night he went to sleep thinking that somehow, they could drive a chaotic system with a signal from an identical system and maybe the two would synchronize. He managed to remember that idea the next day and in the next weeks some simple numerical experiments with iterated maps seemed to confirm that chaotic driving of identical nonlinear systems could cause them to synchronize. But they needed more than just numerical examples featuring simple maps.

Lou and Tom wanted an experimental example of syn- chronous chaos. Tom remembered an analog computer circuit that a professor had demonstrated when he was an undergraduate. The circuit used operational amplifiers, capacitors, and resistors to simulate the equations for a bouncing ball with damping, and the circuit output was displayed on an oscilloscope. He wanted to build an analog computer circuit, and chaotic synchronization gave me a reason. He found in the literature a report of a chaotic circuit developed by Prof. Robert Newcomb of the University of Maryland. ${ }^{185}$ He built a pair of similar circuits: a drive circuit to hide a chaotic signal, and a response circuit which synchronized to the drive circuit in order to extract a message signal that had been added to the driving signal. ${ }^{186} \mathrm{He}$ even used this pair of circuits, along with a digital spectrum analyzer to demonstrate chaotic masking of an information signal in front of NRL's director of research, Dr. Timothy Coffey.

The work on the synchronization of chaotic circuits was still unpublished, but caught the interest of some people from the Space and Naval Warfare Systems Command (SPAWAR) through Dr. Mike Melich of the Naval Postgraduate School in Monterey, California. Tom and Lou were given some funding to pursue our idea of communicating with chaotic signals.

\section{DISCUSSION AND CONCLUSION BY CHRISTOPHE LETELLIER}

From the testimonies in the previous sections, it might be relevant to ask whether the chaos program constitutes a revolution, ${ }^{187}$ a new science, ${ }^{40}$ or a new paradigm in an already established science. A science commonly designates a branch of science as astronomy, physics, chemistry, or mathematics. Chaos is a branch of mathematics with some overlapp with nearly all the other sciences: it is therefore not a new science as supported by Holmes. ${ }^{188}$ A revolution, as it is meant today, designates a radical and sudden change. ${ }^{189}$ Nevertheless, the sudden character is questioned, ${ }^{190}$ mostly because a scientific revolution is the result of a process which is developed over decades if not centuries. It took nearly 20 centuries to switch from the Aristotelician physics to what is called today the classical mechanics. Chaos has clearly not this stature.

More important is the concept of paradigm as promoted by $\mathrm{Kuhn}^{189}$ and which is deliberately defined in an open way. In applied science, a paradigm is based on i) some accepted principles, concepts and rules (invariant set, attractor, Poincaré-Bendixson theorem, Takens theorem, Smale horseshoe, period-doubling cascade, bifurcation, sensitivity to initial conditions...) which provide some permanent solution to a group of outstanding problems, ii) a shared methodology (working in the state space, using numerical simulations, sharing some markers...), and iii) a metaphysics (universality, relationships between mind and matter...). Although with a more or less concious common ground, beliefs and sharing applications to some concrete natural phenomena, scientists working with the chaos paradigm can belong to different schools (statistics versus topology, for instance). Indeed, chaos is a paradigm with its "tacit knowledge" acquired through prac- 
tice and which is not debated. In their history, Aubin and Dahan-Dalmedico oscillate between continuity and rupture to describe the emergence of the nonlinear dynamical systems theory. ${ }^{11}$ To us "epistemological break" as introduced by Bachelard ${ }^{191}$ — that we could define as a new way requiring a new concept or approach to solve a given problem but which is still mainly understood with old concepts - seems more appropriate than "rupture" or revolution as promoted by Kuhn ${ }^{189}$ because all of the current contributors refer to a background, to a scientific heritage; in fact they are quite sensitive to be correctly categorized regarding their academic background. For instance, some of physicists expressed their exact field (radiophysics for AP, condensed matter for LP and TC). All of the contributors reveal that they are highly infuenced by someone - either by his scientific corpus or by his way of thinking (RA inspired by Smale and Thom, DS by Chirikov, RL by Thom and Hénon, LG by Mackey, AP by Rabinovich, LO by Degn and Rössler, CG by Kaufman and Chirikov, UP by Lauterborn, RG by Thom, LP and TC by Grebogi, Yorke and Ott) - or by a key contribution (Lorenz 1963, Ruelle-Takens 1971, among others). Most of them experienced a change of categorization in their activity from a well-defined field (plasma physics, chemistry, radiophysics, condensed matter, engineering) to a field not so clearly identified and recognized by the academic institutions, as already presented in Aubin and Dahan-Dalmedico: nonlinear dynamical systems (NDS) theory or chaos? Very often the two terms are combined, as if it is necessary to clarify some implicit restriction.

Indeed, the NDS theory is characterized by the lack of existence of analytical solutions and, consequently, a qualitative approach is required. This denotes a specific methodology whose foundations date back to Poincaré's works: stability analysis, phase portrait, surface of section, Poincaré map, periodic orbits, etc. Poincaré, who was deeply immersed in the history of his fields, would not deny to qualify his contribution as a epistemological break rather than a rupture or a revolution. He was clearly one of those who are producing better while standing on the shoulders of giants.

From this aspect, all the contributions from the precomputer ages worked within their original scientific discipline: Poincaré, Birkhoff, Lefschetz, Chern, Spanier, and Thom were mostly acting as mathematicians, and were recognized as such. Andronov was working in engineering (anticipating control theory). Nevertheless, a few cases deserve some specific comments. Poincaré is commonly recognized as one of the last "universalists," able to address various problems whose nature was very different. ${ }^{192}$ His epistemological break was to switch from analytical investigations of approximated solutions to differential equations to the qualitative properties of a set of solutions in the state space. René Thom, once he received his Field Medal, felt free to promote a holitistic approach of dynamical processes, evolving strong influences from D'Arcy Thompson ${ }^{193}$ and Paul Dirac ${ }^{194}$ for developing his catastrophe theory: it would be hard to categorize this contribution. Although clearly connected to the NDS theory, as clearly testified in most of the recollections. Edward Lorenz, who was a meteorologist with a strong background in mathematics (his former academic background), switched from mathematics to meteorology during his military service. ${ }^{195}$ Nevertheless, his 1963 contribution ${ }^{10}$ is neither a rupture with other meteorological papers nor in continuation of them since he addressed an old problem - accuracy in weather forecasting - which dates back to Bjerknes ${ }^{196}$ and Richardson, ${ }^{197}$ although the way he treated it was particularly new. It was a more important epistemological break for the NDS theory than for meteorologist (its impact on the field from where it is issued can be compared with Poincaré's méthodes nouvelles in celestial mechanics: ${ }^{8}$ strong in NDS theory, and rather poor in astronomy). The epistemological break is this new combination of mathematical analysis (stability analysis, boundedness, periodic and aperiodic orbits, symbolic analysis of simple maps) with some numerical simulations (state portraits, isopleths, first-return map to a Poincaré section). ${ }^{10}$ The impact of the contributions by Poincare and Lorenz is more important at the intepretation level than for constructing predictive models.

In our view, Lorenz's 1963 contribution is a clear synthesis of the so-called "chaos program" which complements Smale's program as mentioned by RA. Smale's program belongs to the field of mathematics where contributors most often did not use numerical simulations: they propose theorems that they prove analytically. Chaos program is built mostly on numerical data for validating heuristic theory (based on some presupposed assumptions) as experimentalists use their measurements for validating a theory based on presupposed assumptions. Chaos program still belongs to the NDS theory but cannot be considered, stricto sensu, as mathematics. It can also not be consider as physics, engineering, or computer science. This automatically means that the chaos paradigm is part of the NDS theory, issued from mathematics, but it is not recognized as part of mathematics. This explains why, still today, more than 50 years after its birth, "chaos" is a field of scientific activity which is, most often, combined with a classical field (fluid mechanics, radiophysics, plasma, optics, chemistry, ecology, physiology, economy, etc.). As an example, a section in nonlinear Physics from the French Physical Society (SFP) was established as late as in 2021 but, from its name, it is restricted to physics; nonlinear dynamics would have been a far better name.

The first wave of key contributors to the NDS theory from the pre-computer ages - are mostly from mathematics although some of them also contributed to other fields (Poincaré, Thom, Lorenz, Ruelle), and they continued to contribute in mathematics. In the second wave, here associated with those who introduced numerical simulations in their methodology (identified by dashed-dotted lines in Fig. 16), are from various fields (physics, chemistry, engineering, but also mathematics) as shown in Fig. 17 and their works would be more easily categorized in "chaos" rather than in the field of their initial academic background. Some of them even changed the field of their activities (IT from physics to chemistry, LG from chemistry to physiology, $\mathrm{PH}$ from engineering to math). Most of them may eventually published time to time outside from the scientific disciplines. This lack of specific discipline is in fact another source of confusion: the techniques developed can be applied to any system of differential 
equations: it is irrelevant to know from where are coming the equations (until an interpretation is needed). A given scientist can equally contribute to understand the dynamics of an ecosystem and of a pulsating star.

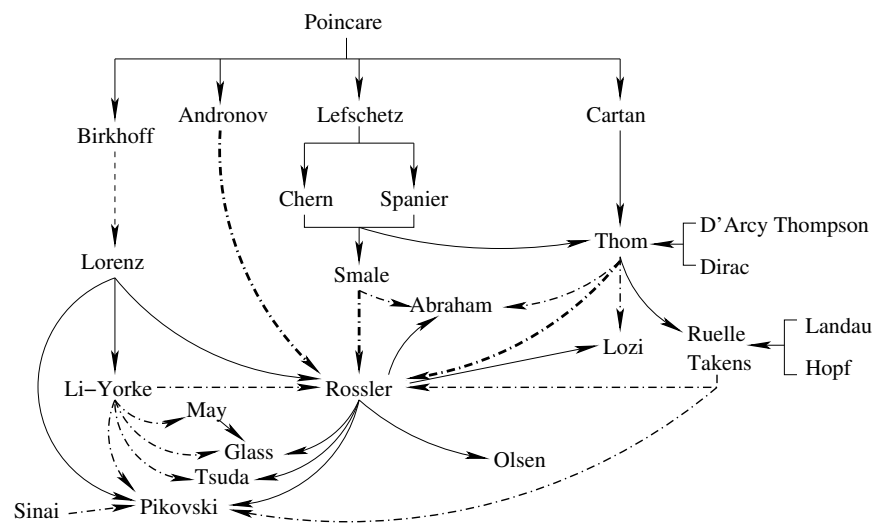

FIG. 16. Some influences between contributors based on some testimonies from the previous sections. The graph is organized for a sake of simplicity. The arrows $(-\cdot-\cdot)$ correspond to the introduction of numerical simualtions. The three bold arrows to Rössler are influences — through direct meetings — before 1975.
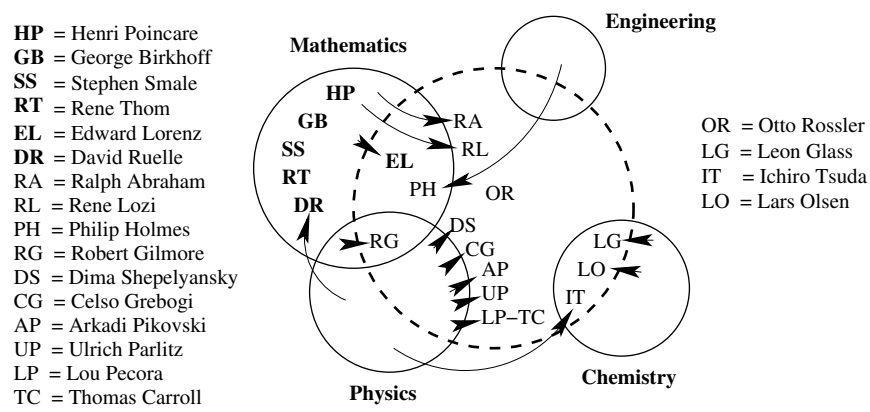

FIG. 17. Distribution of the scientists by their scientific disciplines (mathematics, physics, chemistry and physiology). The central dashed circle represents the branch "chaos" issued from the NDS theory. In bold are the contributors of the first wave who influenced Rössler. Arrows indicate a change in their scientific disciplines.

Among the contributors to the present paper, there is an atypical case: Rössler. Graduated in medicine, he never became a physician, and switched first to behavioral biology (Konrad Lorenz), and to theoretical biology (Robert Rosen). ${ }^{65}$ With his background in electronics as a radio-amateur during his teenage years, he started his career in teaching numerical simulations in chemistry (Tübingen). Before 1976, in the continuation of Rosen's approach, he met Thom, Smale, read Andronov's book, and exchanged ideas with Art Winfree and RA within the paradigm of the NDS theory. He was already publishing in the "chaos" paradigm before 1976 since already using numerical simulations, even though the behaviors investigated were exclusively periodic or quasiperiodic. ${ }^{61,198}$ Earlier contributors did that too as, for instance, Bonhöffer, ${ }^{199}$ FitzHugh, ${ }^{200}$, and Hayashi. ${ }^{201}$ Rössler used only rarely his medical background during the completion of his chaos program between 1976 and 1983: it is thus absent from his "book" written in the early 1980s and only recently published. ${ }^{202}$ In that way, Rössler could be hardly associated with one of the classical scientific disciplines. He was influenced from different scientific disciplines and, in turn, he influenced many peoples working in chemistry, biology and physiology.

In conclusion, chaos is a branch of nonlinear dynamical systems theory which relies on numerical simulations for validating developed rationales (note that we do use neither "theorem" nor "proof"). This is an orphan branch of scientific research in the sense that it can hardly be associated with any classical field. This is eventually due to its abstract nature as well illustrated by Pecora's words:

Of course, this brings a problem at dinner parties when people ask you what you do. Answering Nuclear Physics, Plant Biology, Chemistry, Astronomy, Ecology, will bring at least nods of (a dim) understanding of what you do. But saying Nonlinear Dynamics or, equally the exotic sounding Chaos Theory will bring blank stares and a rush to have the others refresh their drinks.

In spite of these humorous words, there is clearly a corpus of contributions (Poincaré, Smale, Thom, Lorenz, Chirikov, Ruelle-Takens...) from which it emerged. As clearly seen by the different recollections provided, the path to be "initiated" is not unique and, as for every scientific field, there are many ways to contribute to the development of chaos and, more widely, to the nonlinear dynamical systems theory. Each of these paths is based on the interactions and influences of scientists while drinking a coffee or a beer, eating a pizza, or trekking in mountains. This is what web-mediated interactions do not allow and why in-person conferences are absolutely needed.

\section{ACKNOWLEDGMENTS}

L. Glass would like to thank the Natural Sciences and Engineering Research Council (Canada) for its continuous support of curiosity-driven research for over 40 years starting with the events recounted here. He also thanks his colleagues and collaborators including Stuart Kauffman, Rafael Perez, Ronald Shymko, Michael Mackey for their wonderful insights and collaborations during the times recounted here.

R. Gilmore is endebted to the following friends and colleagues, listed in the order encountered on the road described: F. T. Arecchi, L. M. Narducci, J. R. Tredicce, H. G. Solari, E. Eschenazi, G. B. Mindlin, J. L. Birman, J. S. Birman, P. Glorieux, M. Lefranc, C. Letellier, V. Messager, O. E. Rössler, R. Williams.

U. Parlitz would like to thank friends and colleagues who accompanied his first steps into the world of nonlinear phenomena, in particular: U. Dressler, I. Eick, V. Englisch, K. Geist, J. Holzfuss, T. Klinker, W. Knop, A. Kramer, T. Kurz, W. Lauterborn, W. Meyer-Ilse, C. Scheffczyk, E. Suchla, A. Vogel and M. Wiesenfeldt. 
The work by L. Pecora and T. Carroll was supported directly by the Office of Naval Research (ONR) and by ONR through the Naval Research Laboratory's Basic Research Program.

C. Letellier would like to thank Jürgen Kurths for his support to this project.

\section{DATA AVAILABILITY STATEMENT}

This manuscript is a historical review. Data which are quoted in this paper are fully described in the quoted literature.

${ }^{1}$ D. Ruelle and F. Takens, "On the nature of turbulence," Communications in Mathematical Physics 20, 167-192 (1971).

${ }^{2}$ T.-Y. Li and J. A. Yorke, "Period three implies chaos," The American Mathematical Monthly 82, 985-992 (1975).

${ }^{3}$ O. E. Rössler, "Chaotic behavior in simple reaction system," Zeitschrift für Naturforschung A 31, 259-264 (1976).

${ }^{4}$ O. E. Rössler, "Different types of chaos in two simple differential equations," Zeitschrift für Naturforschung A 31, 1664-1670 (1976).

${ }^{5}$ O. E. Rössler, "An equation for continuous chaos," Physics Letters A 57, 397-398 (1976).

${ }^{6}$ O. E. Rössler, "Chemical turbulence: Chaos in a simple reaction-diffusion system," Zeitschrift für Naturforschung A 31, 1168-1172 (1976).

${ }^{7} \mathrm{H}$. Poincaré, "Sur le problème des trois corps et les équations de la dynamique," Acta mathematica 13, 1-270 (1890).

${ }^{8} \mathrm{H}$. Poincaré, Les méthodes nouvelles de la mécanique céleste III (GauthierVilard, Paris, 1899).

${ }^{9}$ G. D. Birkhoff, Dynamical Systems (American Mathematical Society, 1927).

${ }^{10}$ E. N. Lorenz, "Deterministic nonperiodic flow," Journal of the Atmospheric Sciences 20, 130-141 (1963).

${ }^{11}$ D. Aubin and A. D. Dalmedico, "Writing the history of dynamical systems and chaos: Longue durée and revolution, disciplines and cultures," Historia Mathematica 29, 273-339 (2002).

${ }^{12}$ V. V. Nemytskii and V. V. Stepanov, Qualitative Theory of Differential Equations (Princeton University Press, 1960) translation of the 2nd Russian edition, 1949.

${ }^{13}$ E. N. Lorenz, The essence of chaos (University of Washington Press, 1993).

${ }^{14}$ R. H. Abraham and Y. Ueda, The chaos avant-garde: Memories of the early days of chaos theory (World Scientific Publishing, Singapore, 2000).

${ }^{15}$ S. Lefschetz, Lectures on Differential Equations (Princeton University Press, 1946).

${ }^{16} \mathrm{~S}$. Smale, The mathematics of time: Essays on dynamical systems, economic processes, and related topics (Springer-Verlag, New York, 1980).

${ }^{17}$ M. M. Peixoto, "Structural stability on two-dimensional manifolds," Topology 1, 101-120 (1962).

${ }^{18} \mathrm{~S}$. Smale, "Generalized Poincaré's conjecture in dimensions greater than four," Annals of Mathematics 74, 391-406 (1961).

${ }^{19} \mathrm{R}$. Thom, "Un lemme sur les applications différentiables," Boletín de la Sociedad Matemática Mexicana 1, 59-71 (1956).

${ }^{20}$ S. Smale, "Differentiable dynamical systems. I Diffeormorphisms," Bulletin of American Mathematical Society 73, 747-817 (1967).

${ }^{21}$ G. D. Birkhoff and P. A. Smith, "Structure analysis of surface transformations," Journal de Mathématiques Pures et Appliquées IX, 7, 345-380 (1928).

${ }^{22}$ H. Poincaré, "Sur les courbes définies par les équations différentielles (3)," Journal de Mathématiques Pures et Appliquées, IV 1, 167-244 (1885).

${ }^{23}$ G. D. Birkhoff, "Surface transformations and their dynamical applications," Acta Mathematica 43, 1-119 (1922).

${ }^{24}$ M. Morse, "The critical points of a function of $n$ variables," Transactions of the American Mathematical Society 33, 72-91 (1931).

${ }^{25}$ A. A. Andronov and L. S. Pontryagin, "Rough systems (in russian)," Doklady Akademii Nauk SSSR 14, 247-250 (1937).
${ }^{26} \mathrm{R}$. Thom, "Sur une partition en cellules associée à une function sur une variété," Comptes-Rendus de l'Académie des Sciences 228, 973-975 (1949).

${ }^{27}$ L. E. El'sgol'ts, "An estimate for the number of singular points of a dynamical system defined on a manifold," Matematicheskii Sbornik 26, 215-223 (1950), translated for the American Mathematical Society, 1952.

${ }^{28}$ G. Reeb, "Sur certaines propriétés topologiques des trajectoires des systèmes dynamiques," Mémoires de l'Académie Royale de Belgique: Classe des Sciences 27, 9 (1952).

${ }^{29}$ M. M. Peixoto, "On structural stability," Annals of Mathematics 69, 199222 (1959).

${ }^{30}$ R. H. Abraham and J. E. Marsden, Foundations of Mechanics: a mathematical exposition of classical mechanics (W. A. Benjamin, Reading, MA, 1967).

${ }^{31}$ R. H. Abraham and J. W. Robbin, Transversal mappings and flows (W. A. Benjamin, Reading, MA, 1967).

${ }^{32} \mathrm{R}$. Thom, Structural stability and morphogenesis: An outline of a general theory of models (W. A. Benjamin, New York, 1973) translated from the French by David Fowler.

${ }^{33}$ C. E. Zeeman, Catastrophe theory: Selected papers, 1972-1977 (AddisonWesley, Reading, Massachusetts, 1977).

${ }^{34}$ S.-S. Chern and S. Smale, eds., Global analysis, Proceedings of Symposia in Pure Mathematics No. 14 (American Mathematical Society, Providence, RI, 1970) (Berkeley, CA, 1-26 July 1968).

${ }^{35}$ D. Ruelle, "Strange attractors," The Mathematical Intelligencer 2, 126-137 (1980).

${ }^{36}$ Y. Ueda, "Some problems in the theory of nonlinear oscillations (PH.D. thesis, 1965)," in The road to chaos (Aerial Press, 1992).

${ }^{37} \mathrm{C}$. Mira, "étude de la frontière de stabilité d'un point double stable d'une récurrence non linéaire autonome du deuxième ordre," in Proceedings of the IFAC Symposium on Pulse-rate and Pulse-number Signals in Automatic Control (1968) pp. D 43-7II.

${ }^{38}$ I. Gumowski and C. Mira, "Sensitivity problems related to certain bifurcations in non-linear recurrence relations," Automatica 5, 303-317 (1969).

${ }^{39}$ R. H. Abraham and C. D. Shaw, Dynamics the geometry of behavior (Aerial Press, Santa Cruz, CA, 1981-1988).

${ }^{40}$ J. Gleick, Chaos: Making a new science (Viking Press, New York, 1987).

${ }^{41}$ J. P. Crutchfield, J. D. Farmer, N. H. Packard, and R. S. Shaw, "Chaos," Scientific American 255, 46-57 (1986).

${ }^{42}$ D. Shepelyansky, "Boris Valerianovich Chirikov," Scholarpedia 3, 6628 (2008).

${ }^{43}$ D. L. Shepelyansky, "Website dedicated to Boris Chirikov," www.quantware.ups-tlse.fr/chirikov/, last access, August 2020.

${ }^{44}$ B. V. Chirikov, "Resonance processes in magnetic traps," Atomnaya Energiya 6, 630 (1959), in Russian.

${ }^{45}$ B. V. Chirikov, "Resonance processes in magnetic traps," The Soviet Journal of Atomic Energy 6, 464-470 (1960).

${ }^{46} \mathrm{~B}$. V. Chirikov, "Research concerning the theory of non-linear resonance and stochasticity," Tech. Rep. IYAF-267-TRANS-E (Institute of Nuclear Physics, Novosibirsk, 1969) translated at CERN from the Russian, CERNTrans-71-40, 1971.

${ }^{47}$ B. V. Chirikov, "A universal instability of many-dimensional oscillator systems," Physics Reports 52, 263 - 379 (1979).

${ }^{48}$ B. V. Chirikov, F. M. Izrailev, and D. L. Shepelyansky, "Dynamical stochasticity in classical and quantum mechanics," Soviet Scientific Reviews C 2, 209-267 (1981).

${ }^{49}$ B. V. Chirikov, F. M. Izrailev, and D. L. Shepelyansky, "Quantum chaos: Localization vs. ergodicity,” Physica D 33, 77-88 (1988).

${ }^{50} \mathrm{~B}$. V. Chirikov, "Some numerical experiments with a nonlinear mapping: stochastic component," (Colloques Internationaux du CNRS, Toulouse, 1973) pp. 409-428.

${ }^{51}$ B. V. Chirikov and F. M. Izraelev, "Degeneration of turbulence in simple systems," Physica D 2, 30 - 37 (1981).

${ }^{52}$ K. Frahm and D. Shepelyansky, "Ulam method for the Chirikov standard map," European Physical Journal B 76, 57-68 (2010).

${ }^{53}$ G. Casati, B. V. Chirikov, F. M. Izraelev, and J. Ford, "Stochastic behavior of a quantum pendulum under a periodic perturbation," Lecture Notes in Physics 93, 334-352 (1979).

${ }^{54}$ N. Rashevksy, Mathematical Biophysics: Physico-Mathematical Foundations of Biology (University of Chicago Press, 1938). 
${ }^{55}$ N. Rashevksy, Some Medical Aspects of Mathematical Biology (C. C. Thomas, Springfield, IL, 1964).

${ }^{56} \mathrm{~N}$. Rashevksy, "Outline of a unified approach to physics, biology and sociology," Bulletin of Mathematical Biophysics 31, 159-198 (1969).

${ }^{57}$ R. Rosen, Dynamical Systems Theory in Biology (Wiley Interscience, New York, 1970).

${ }^{58}$ A. A. Andronov, A. A. Vitt, and S. E. Khaikin, Teoriya kolebanii (Fizmatgiz, Moscow, 1959) Theory of oscillators, Pergamon Press, 1966.

${ }^{59}$ O. E. Rössler, "Grundschaltungen von flüssigen Automaten und Reaktionssystemen," Zeitschrift für Naturforschung B 27, 333-343 (1972).

${ }^{60}$ W. Huber, G. Simon, and H. Kuhn, "Analogiebetrachtungen und Analogrechner zur Behandlung der Korrelation von $\pi$-Elektronen (1)," Zeitschrift für Naturforschung A 17, 99-114 (1962).

${ }^{61}$ O. E. Rössler, "Repetitive hard bifurcation in a homogeneous reaction system," in Analysis and Simulations of Biochemical Systems, edited by H. C. Hemkers and B. Hess (Amsterdam, North-Holland, 1972) pp. 91-102, proceedings of the 8th FEBS meeting.

${ }^{62}$ A. T. Winfree, "Spiral waves of chemical activity," Science 175, 634-636 (1972).

${ }^{63}$ A. T. Winfree, "Scroll-shaped waves of chemical activity in three dimensions," Science 181, 937-939 (1973).

${ }^{64}$ O. E. Rössler, "Chemical automata in homogeneous and reaction-diffusion kinetics," Lecture Notes in Biomathematics 4, 399-418 (1974).

${ }^{65} \mathrm{C}$. Letellier and V. Messager, "Influences on Otto E. Rössler's earliest paper on chaos," International Journal of Bifurcation \& Chaos 20, 3585-3616 (2010).

${ }^{66}$ O. E. Rössler, "Continuous chaos," (Springer-Verlag, Berlin, Germany, 1977) pp. 174-183.

${ }^{67}$ J. Crutchfield, D. Farmer, N. Packard, R. Shaw, G. Jones, and R. Donnelly, "Power spectral analysis of a dynamical system," Physics Letters A 76, 14 (1980).

${ }^{68}$ https : //www . youtube . com/watch?v=Tmmdg2P1RIM.

${ }^{69}$ E. C. Zeeman, "Duffing's equation in brain modelling," Bulletin of the Institute of Mathematics and Applications 12, 207-214 (1976)

${ }^{70}$ P. J. Holmes and D. A. Rand, "The bifurcations of Duffing's equation: An application of catastrophe theory," Journal of Sound and Vibration 44, 237 -253 (1976).

${ }^{71}$ P. J. Holmes and D. A. Rand, "Bifurcations of the forced van der Pol oscillator," Quarterly of Applied Mathematics 35, 495-509 (1978).

${ }^{72} \mathrm{P}$. Holmes and D. Rand, "Phase portraits and bifurcations of the non-linear oscillator: $\ddot{x}+\left(\alpha+\gamma x^{2}\right) \dot{x}+\beta x+\delta x^{3}=0$," International Journal of NonLinear Mechanics 15, 449-458 (1980).

${ }^{73}$ F. Takens, "Singularities of vector fields," Publications Mathématiques de l'Institut des Hautes Études Scientifique 43, 47-100 (1974).

${ }^{74} \mathrm{~F}$. Takens, "Forced oscillations and bifurcations," Communications of the Mathematics Institute 3, 1-59 (1974).

${ }^{75}$ M. L. Cartwright, "Forced oscillations in nonlinear systems," in Contributions to the Theory of Nonlinear Oscillations, Vol. 20 (Princeton University Press, Princeton, 1950) pp. 149-242.

76 "Bifurcation theory and applications in scientific disciplines," in Annals of the New York Academy of Sciences, Vol. 316, edited by O. Gurel and O. E. Rössler (1979) pp. 1-686

77 "Nonlinear dynamics," in Annals of the New York Academy of Sciences, Vol. 357, edited by R. H. G. Helleman (1980) pp. 1-505.

78 "New approaches to nonlinear problems in dynamics," in Proceedings of the Engineering Foundation Conference, edited by P. Holmes, Asilomar CA, December 9-14, 1979 (SIAM Publications, Philadelphia, 1980).

${ }^{79} \mathrm{R}$. Lozi, "Modèles mathématiques simples et consistants pour l'étude de quelques systèmes dynamiques expérimentaux," (1983), Thèse d'État, University of Nice, France.

${ }^{80} \mathrm{H}$. Kawakami and R. Lozi, "Switched dynamical systems - dynamics of a class of circuits with switch," in Avanced Series in Dynamical Systems, Vol. 11, edited by S. Ushiki (World Scientific Publishing, Singapore, 1992) pp. 39-58.

${ }^{81}$ H. Kawakami and R. Lozi, "Switched dynamical systems - a switch defines semi-flows on a pair of half planes," in Proceedings of the RIMS Conference, Vol. 804 (RIMS Kokyuroku, Japan, 1992) pp. 38-54, in Japanese.

${ }^{82}$ O. E. Rössler, "Continuous chaos: four prototype equations," Annals of the New York Academy of Sciences 316, 376-392 (1979).
${ }^{83}$ O. E. Rössler, "Chaotic oscillations: an example of hyperchaos," Lectures in Applied Mathematics 17, 141-156 (1979).

${ }^{84}$ O. E. Rössler, "An equation for hyperchaos," Physics Letters A 71, 155157 (1979).

${ }^{85}$ R. Lozi, "Dimensional bifurcations between thread and sheet strange attractor," in Théorie de l'itération et ses applications, Vol. 322, edited by R. Thibault (Colloques Internationaux du CNRS, 1982) pp. 145-152.

${ }^{86} \mathrm{R}$. Lozi, Analyse numérique de certains problèmes de bifurcation, Ph.D. thesis, University of Nice, France (1975).

${ }^{87}$ H. Poincaré, "Sur l'équilibre d'une masse fluide animée d'un mouvement de rotation," Comptes Rendus de l'Académie des Sciences 100, 346-348 (1885).

${ }^{88}$ N. Bourbaki, Éléments de mathématiques (Masson, 1959-2019) collective work.

${ }^{89} \mathrm{G}$. Iooss, "Bifurcation des solutions périodiques de certains problèmes d'évolution," Comptes Rendus de l'Académie des Sciences I 273, 624627 (1971)

${ }^{90} \mathrm{G}$. Iooss and R. Lozi, "Convection entre deux plaques planes en rotation et effet dynamo résultant d'une bifurcation secondaire," Journal de Physique - Colloques 39, C5-15-C5-16 (1977).

${ }^{91}$ D. Ruelle, "Dynamical systems with turbulent behavior," Lecture Notes in Physics 80, 341-360 (1978), International Mathematics Physics Conference, Roma 1977.

${ }^{92}$ M. Hénon, "A two-dimensional mapping with a strange attractor," Communications in Mathematical Physics 50, 69-77 (1976).

${ }^{93}$ E. Zeraoulia, Lozi mappings - Theory and applications (CRC Press, 2013).

${ }^{94}$ R. Lozi, "Un attracteur étrange (?) du type attracteur de Hénon," Journal de Physique 39, C5-9-C5-10 (1978).

${ }^{95}$ M. Misiurewicz, "Strange attractors for the Lozi mappings," Annals of the New York Academy of Sciences 357, 348-358 (1980).

${ }^{96}$ D. Ruelle, "Measure describing a turbulent flow," Annals of the New-York Academy of Sciences 357, 1-9 (1980).

${ }^{97}$ C. Vidal, J.-C. Roux, S. Bachelart, and A. Rossi, "Experimental study of the transition to turbulence in the Belousov-Zhabotinsky reaction," Annals of the New-York Academy of Sciences 357, 377-396 (1980).

${ }^{98}$ S. A. Kauffman, "Metabolic stability and epigenesis in randomly constructed genetic nets," Journal of Theoretical Biology 22, 437-467 (1969).

${ }^{99}$ L. Glass and S. A. Kauffman, "Co-operative components, spatial localization and oscillatory cellular dynamics," Journal of Theoretical Biology 34, 219-237 (1972).

${ }^{100}$ L. Glass and S. A. Kauffman, "The logical analysis of continuous, nonlinear biochemical control networks," Journal of theoretical Biology 39, 103-129 (1973).

${ }^{101}$ M. J. Feigenbaum, "Universal behavior in nonlinear systems," Los Alamos Science , 4-27 (1980).

${ }^{102}$ M. C. Mackey and L. Glass, "Oscillation and chaos in physiological control systems," Science 197, 287-289 (1977).

${ }^{103}$ L. Glass and M. C. Mackey, "Pathological conditions resulting from instabilities in physiological control system," Annals of the New York Academy of Sciences 316, 214-235 (1979).

${ }^{104}$ R. M. May, "Simple mathematical models with very complicated dynamics," Nature 261, 459-467 (1976).

${ }^{105}$ R. M. May and G. F. Oster, "Bifurcations and dynamic complexity in simple ecological models," The American Naturalist 110, 573-599 (1976).

${ }^{106} \mathrm{O}$. Gurel and O. E. Rössler, Bifurcation theory and appliations in scientific disciplines, Annals of the New York Academy of Sciences, Vol. 316 (New York Academy of Sciences, 1979) papers from the conference held on October 31-November 4, 1977.

${ }^{107}$ T. A. Bass, The eudaemonic pie (Houghton Mifflin Harcourt, 1985).

${ }^{108}$ N. H. Packard, J. P. Crutchfield, J. D. Farmer, and R. S. Shaw, "Geometry from a time series," Phys. Rev. Lett. 45, 712-716 (1980).

${ }^{109}$ J. D. Farmer, "Chaotic attractors of an infinite-dimensional dynamical system," Physica D 4, 366-393 (1982).

${ }^{110}$ L. Glass and M. Mackey, "Mackey-Glass equation," Scholarpedia 5, 6908 (2010).

${ }^{111}$ A. Gaponov, L. Ostrovskii, and M. I. Rabinovich, "One-dimensional waves in disperse nonlinear systems," Radiophysics and Quantum Electronics 13, 121-161 (1970). 
${ }^{112}$ B. B. Kadomtsev and V. I. Karpman, "Nonlinear waves," Soviet Physics Uspekhi 14, 40-60 (1971).

${ }^{113} \mathrm{~J}$. B. McLaughlin and P. C. Martin, "Transition to turbulence of a statically stressed fluid," Physical Review Letters 33, 1189-1192 (1974).

${ }^{114}$ G. M. Zaslavskiı̌ and B. V. Chirikov, "Stochastic instability of non-linear oscillations," Soviet Physics Uspekhi 14, 549-568 (1972).

${ }^{115}$ Y. G. Sinai, "On the foundations of the ergodic hypothesis for a dynamical system of statistical mechanics," Doklady Akademii Nauk SSSR 153, 1261-1264 (1963).

${ }^{116}$ Y. G. Sinai, "Dynamical systems with elastic reflections. Ergodic properties of dispersing billiards," Russian Mathematical Surveys 25, 137-189 (1970).

${ }^{117}$ S. I. Vyshkind and M. I. Rabinovich, "The mechanism for phase stochastization and the structure of wave turbulence in dissipative media," Soviet Journal of Experimental and Theoretical Physics 44, 292-299 (1976).

${ }^{118}$ A. S. Pikovski, M. I. Rabinovich, and V. Y. Trakhtengerts, "Onset of stochasticity in decay confinement of parametric instability," Zhurnal Eksperimentalnoi i Teoreticheskoi Fiziki 74, 1366-1374 (1978).

${ }^{119}$ A. S. Pikovski and M. I. Rabinovich, "A simple autogenerator with stochastic behavior," Dokladi Akademii Nauk SSSR 239, 301-304 (1978).

${ }^{120} \mathrm{H}$. Degn, "Effect of bromine derivatives of malonic acid on the oscillating reaction of malonic acid, cerium ions and bromate," Nature 213, 589-590 (1967).

${ }^{121} \mathrm{H}$. Degn, "Bistability caused by substrate inhibition of peroxidase in an open reaction system," Nature 217, 1047-1050 (1968).

${ }^{122}$ H. Degn, L. F. Olsen, and J. W. Perram, "Bistability, oscillation, and chaos in an enzyme reaction," Annals of the New York Academy of Sciences 316, 623-637 (1979).

${ }^{123}$ L. F. Olsen and H. Degn, "Chaos in an enzyme reaction," Nature 267, 177178 (1977)

${ }^{124}$ R. A. Schmitz, K. R. Graziani, and J. L. Hudson, "Experimental evidence of chaotic states in the Belousov-Zhabotinskii reaction," The Journal of Chemical Physics 67, 3040-3044 (1977).

${ }^{125}$ O. E. Rössler and K. Wegmann, "Chaos in the Zhabotinskii reaction," Nature 271, 89-90 (1978).

${ }^{126}$ F. Takens, "Detecting strange attractors in turbulence," Lectures Notes in Mathematics 898, 366-381 (1981).

${ }^{127}$ J. L. Hudson, M. Hart, and D. Marinko, "An experimental study of multiple peak periodic and nonperiodic oscillations in the BelousovZhabotinskii reaction," The Journal of Chemical Physics 71, 1601-1606 (1979).

${ }^{128}$ J. Roux, A. Rossi, S. Bachelart, and C. Vidal, "Experimental observations of complex dynamical behavior during a chemical reaction," Physica D 2, $395-403$ (1981)

${ }^{129} \mathrm{~K}$. Tomita and I. Tsuda, "Chaos in the Belousov-Zhabotinsky reaction in a flow system," Physics Letters A 71, 489-492 (1979).

${ }^{130} \mathrm{~K}$. Tomita and I. Tsuda, "Towards the interpretation of Hudson's experiment on the Belousov-Zhabotinsky reaction: Chaos due to delocalization," Progress of Theoretical Physics 64, 1138-1160 (1980).

${ }^{131} \mathrm{~K}$. Wegmann and O. E. Rössler, "Different kinds of chaotic oscillations in the Belousov-Zhabotinskii reaction," Zeitschrift für Naturforschung A 33, 1179-1183 (1978).

${ }^{132}$ R. J. Field and R. M. Noyes, "Oscillations in chemical systems. iv. Limit cycle behavior in a model of a real chemical reaction," The Journal of Chemical Physics 60, 1877-1884 (1974).

${ }^{133}$ B. R. Hunt and J. A. Yorke, "Maxwell on chaos," Nonlinear Science Today 3, 1-4 (1993).

${ }^{134}$ F. Diacu and P. Holmes, Celestial Encounters: The Origins of Chaos and Stability (Princeton University Press, 1996).

${ }^{135} \mathrm{~J}$. Barrow-Green, Poincaré and the Three Body Problem, History of Mathematics, Vol. 11 (American Mathematical Society, 1997).

${ }^{136}$ V. I. Arnol'd, Mathematical Methods of Classical Mechanics, Graduate Texts in Mathematics, Vol. 60 (Springer-Verlag, New York, 1989) translated by A. Weinstein and K. Vogtmann.

${ }^{137}$ S. W. McDonald and A. N.Kaufman, "Spectrum and eigenfunctions for a Hamiltonian with stochastic trajectories," Physical Review Letters 42, 1189-1191 (1979).

${ }^{138}$ M. V. Berry, "Forms of light," The Sciences 19, 18-20 (1979).

${ }^{139}$ R. Thom, "Structural stability, catastrophe theory, and applied mathematics," SIAM Review 19, 189-201 (1977).
${ }^{140} \mathrm{C}$. Grebogi, E. Ott, and J. A. Yorke, "Metamorphoses of basin boundaries in nonlinear dynamical systems," Physical Review Letters 56, 1011-1014 (1986).

${ }^{141}$ T. Shinbrot, C. Grebogi, J. Wisdom, and J. A. Yorke, "Chaos in a double pendulum," American Journal of Physics 60, 491-499 (1992).

${ }^{142} \mathrm{C}$. Grebogi, E. Ott, and J. A. Yorke, "Chaos, strange attractors, and fractal basin boundaries in nonlinear dynamics," Science 238, 632-638 (1987).

${ }^{143} \mathrm{H}$. Haken, Erfolgsgeheimnisse der Natur - Synergetik: Die Lehre vom Zusammenwirken (Deutsche Verlags-Anstalt, Stuttgart, 1981).

${ }^{144}$ I. Prigogine and I. Stengers, Dialog mit der Natur - Neue Wege naturwissenschaftlichen Denkens (R. Piper \& Co. Verlag, München Zürich, 1981).

${ }^{145} \mathrm{M}$. Eigen and R. Winkler, Das Spiel - Naturgesetze steuern den Zufall (R. Piper \& Co. Verlag, München Zürich, 1975).

${ }^{146} \mathrm{M}$. Eigen and R. Winkler, The Laws of the Game: How The Principles of Nature Govern Chance (Princeton University Press, 1975).

${ }^{147}$ W. Lauterborn, "Numerical investigation of nonlinear oscillations of gas bubbles in liquids," The Journal of the Acoustical Society of America 59, 283-293 (1976).

${ }^{148}$ W. Lauterborn and E. Cramer, "Subharmonic route to chaos observed in acoustics," Physical Review Letters 47, 1445-1448 (1981).

${ }^{149} \mathrm{~J}$. Guckenheimer and P. J. Holmes, Nonlinear oscillations, dynamical systems, and bifurcations of vector fields, Applied Mathematical Sciences, Vol. 42 (Springer-Verlag, New York Heidelberg Berlin, 1983).

${ }^{150} \mathrm{U}$. Parlitz and W. Lauterborn, "Superstructure in the bifurcation set of the Duffing equation $\ddot{x}+d \dot{x}+x+x^{3}=f \cos (\omega t)$," Physics Letters A 107, 351355 (1985).

${ }^{151}$ T. Uezu and Y. Aizawa, "Topological Character of a Periodic Solution in Three-Dimensional Ordinary Differential Equation System," Progress of Theoretical Physics 68, 1907-1916 (1982).

${ }^{152} \mathrm{U}$. Parlitz and W. Lauterborn, "Resonances and torsion numbers of driven dissipative nonlinear oscillators," Zeitschrift für Naturforschung A 41, 605- 614 (1986)

${ }^{153}$ U. Parlitz, V. Englisch, C. Scheffczyk, and W. Lauterborn, "Bifurcation structure of bubble oscillators," The Journal of the Acoustical Society of America 88, 1061-1077 (1990).

${ }^{154}$ C. Scheffczyk, U. Parlitz, T. Kurz, W. Knop, and W. Lauterborn, "Comparison of bifurcation structures of driven dissipative nonlinear oscillators," Physical Review A 43, 6495-6502 (1991).

${ }^{155}$ U. Parlitz and W. Lauterborn, "Period-doubling cascades and devil's staircases of the driven van der Pol oscillator," Physical Review A 36, 14281434 (1987).

${ }^{156}$ M. L. Cartwright and J. E. Littlewood, "On non-linear differential equations of the second order, I," Journal of the London Mathematical Society 20, 180 (1945)

${ }^{157}$ R. Gilmore, C. M. Bowden, and L. M. Narducci, "Classical-quantum correspondence for multilevel systems," Physical Review A 12, 1019-1031 (1975).

${ }^{158} \mathrm{H}$. Haken, "Analogy between higher instabilities in fluids and lasers," Physics Letters A 53, 77-78 (1975).

${ }^{159} \mathrm{C}$. O. Weiss and W. Klische, "On observability of Lorenz instabilities in lasers," Optics Communications 51, 47-48 (1984).

${ }^{160}$ C. O. Weiss and J. Brock, "Evidence for Lorenz-type chaos in a laser," Physical Review Letters 57, 2804-2806 (1986).

${ }^{161}$ T. Poston and I. Stewart, Catastrophe theory and its applications (Pitman, London, 1978).

${ }^{162}$ R. Gilmore, Catastrophe theory for scientists and engineers (Wiley, New York, 1981).

${ }^{163}$ K. G. Wilson, "Renormalization group and critical phenomena. i. Renormalization group and the Kadanoff scaling picture," Physical Review B 4, 3174-3183 (1971).

${ }^{164}$ K. G. Wilson, "Renormalization group and critical phenomena. ii. phasespace cell analysis of critical behavior," Physical Review B 4, 3184-3205 (1971).

${ }^{165}$ C. Tresser and P. Coullet, "Itérations d'endomorphismes et groupe de renormalization," Comptes-Rendus de l'Académie des Sciences (Paris) 287A, 577-580 (1978).

${ }^{166} \mathrm{P}$. Coullet and C. Tresser, "Itération d'endomorphismes et groupe de renormalization," Journal de Physique Colloques 39, C5-25-C5-28 (1978).

${ }^{167}$ M. J. Feigenbaum, "Quantitative universality for a class of nonlinear transformations," Journal of Statistical Physics 19, 25-52 (1978). 
${ }^{168}$ M. J. Feigenbaum, "The universal metric properties of nonlinear transformations," Journal of Statistical Physics 21, 669-706 (1979).

${ }^{169}$ P. Cvitanović, Universality in chaos (Adam Hilger, Boston, 1984).

${ }^{170}$ F. T. Arecchi, R. Meucci, G. Puccioni, and J. Tredicce, "Experimental evidence of subharmonic bifurcations, multistability, and turbulence in a $q$-switched gas laser," Physical Review Letters 49, 1217-1220 (1982).

${ }^{171}$ I. I. Matorin, A. S. Pikovskiǔ, and Y. I. Khanin, "Multistability and autostochasticity in a laser with a delayed-response active medium subjected to periodic loss modulation," Soviet Journal of Quantum Electronics 14, 1401-1405 (1984).

${ }^{172}$ H. G. Solari, E. Eschenazi, R. Gilmore, and J. R. Tredicce, "Influence of coexisting attractors on the dynamics of a laser system," Optics Communications 64, 49-53 (1987).

${ }^{173}$ H. G. Solari and R. Gilmore, "Relative rotation rates for driven dynamical systems," Physical Review A 37, 3096-3109 (1988).

${ }^{174}$ H. G. Solari and R. Gilmore, "Organization of periodic orbits in the driven Duffing oscillator," Physical Review A 38, 1566-1572 (1988).

${ }^{175}$ J. Birman and R. F. Williams, "Knotted periodic orbits in dynamical systems I. Lorenz's equations," Topology 22, 47-82 (1983).

${ }^{176}$ J. Birman and R. F. Williams, "Knotted periodic orbits in dynamical systems II. Knot holders for fibred knots," Contemporary Mathematics 20, 1-60 (1983).

${ }^{177}$ G. B. Mindlin, X.-J. Hou, H. G. Solari, R. Gilmore, and N. B. Tufillaro, "Classification of strange attractors by integers," Physical Review Letters 64, 2350-2353 (1990).

${ }^{178}$ G. B. Mindlin, H. G. Solari, M. A. Natiello, R. Gilmore, and X.-J. Hou, "Topological analysis of chaotic time series data from the BelousovZhabotinskii reaction," Journal of Nonlinear Science 1, 147-173 (1991).

${ }^{179}$ R. Gilmore, R. Vilaseca, R. Corbalán, and E. Roldán, "Topological analysis of chaos in the optically pumped laser," Physical Review E 55, 24792487 (1997).

${ }^{180}$ R. Gilmore, "Topological analysis of chaotic dynamical systems," Reviews of Modern Physics 70, 1455-1529 (1998).

${ }^{181}$ C. D. Jeffries, "Chaotic dynamics of instabilities in solids," Physica Scripta T9, 11-26 (1985).

${ }^{182}$ C. Grebogi, E. Ott, and J. A. Yorke, "Super persistent chaotic transients," Ergodic Theory and Dynamical Systems 5, 341-372 (1985).

${ }^{183}$ T. L. Carroll, L. M. Pecora, and F. J. Rachford, "Chaotic transients and multiple attractors in spin-wave experiments," Physical Review Letters 59, 2891-2894 (1987).
${ }^{184}$ L. M. Pecora and T. L. Carroll, "Synchronization in chaotic systems," Physical Review Letters 64, 821-824 (1990).

${ }^{185}$ R. Newcomb and S. Sathyan, “An RC Op Amp chaos generator," IEEE Transactions on Circuits and Systems 30, 54-56 (1983).

${ }^{186}$ T. L. Carroll and L. M. Pecora, "Synchronizing chaotic circuits," IEEE Transactions on Circuits and Systems 38, 453-456 (1991).

${ }^{187}$ D. Parrochia, Les grandes révolutions scientifiques du XXe siècle (Presses Universitaires de France, Paris, 1997).

${ }^{188} \mathrm{P}$. Holmes, "Poincaré, celestial mechanics, dynamical-systems theory and "chaos"," Physics Reports 193, 137-163 (1990).

${ }^{189}$ T. S. Kuhn, The structure of scientific revolutions, 2 nd ed. (The University of Chicago Press, Chicago, 1970).

${ }^{190}$ I. B. Cohen, "The eighteenth-century origins of the concept of scientific revolution," Journal of the History of Ideas , 257-288 (1976).

${ }^{191}$ G. Bachelard, The Formation of the Scientific Mind: A Contribution to a Psychoanalysis of Objective Knowledge (Beacon Press, 1986) english translation from the French, Vrin (Paris), 1938.

${ }^{192}$ J. Mawhin, "Henri poincaré. a life in the service of science," Notices of the American Mathematical Society 52, 1036-1044 (2005).

${ }^{193} \mathrm{D}^{\prime}$ Arcy Thompson, On growth and form (Cambridge University Press, 1917).

${ }^{194}$ P. A. M. Dirac, The principles of quantum mechanics (Clarendon Press, Oxford, 1930).

${ }^{195}$ E. N. Lorenz, "The bulletin interviews professor edward n. lorenz," World Meteorological Organization Bulletin 45, 111-120 (1996).

${ }^{196}$ V. Bjerknes, "Das Problem der Wettervorhersage, betrachtet vom Standpunkte der Mechanik und der Physik," Meteorologische Zeitschrift 21, 1-7 (1904).

${ }^{197}$ L. F. Richardson, Weather Prediction by Numerical Process (Cambridge University Press, Cambridge, England, 1922).

${ }^{198}$ O. E. Rössler, "A multivibrating switching network in homogeneous kinetics," Bulletin of Mathematical Biology 37, 181-192 (1975).

${ }^{199}$ K. F. Bonhöffer, "Activation of passive iron as a model for the excitation of nerve," Journal of General Physiology 32, 69-91 (1948).

${ }^{200} \mathrm{R}$. FitzHugh, "Impulses and physiological states in theoretical models of nerve membrane," Biophysical Journal 1, 445-466 (1961).

${ }^{201}$ C. Hayashi, Nonlinear oscillations in physical systems (McGraw-Hill, 1964).

${ }^{202}$ O. E. Rössler and C. Letellier, Chaos: The world of nonperiodic oscillations (Springer Nature, Switzerland AG, 2020). 